\title{
The Immunoenhancement Effects of Polyethylenimine-Modified Chinese Yam Polysaccharide-Encapsulated PLGA Nanoparticles as an Adjuvant
}

This article was published in the following Dove Press journal:

International Journal of Nanomedicine

\author{
Yue Zhang ${ }^{1,2}$ \\ Pengfei Gu ${ }^{1,2}$ \\ Adelijiang Wusiman ${ }^{1,2}$ \\ Shuwen $\mathrm{Xu}^{1,2}$ \\ Haiyu $\mathrm{Ni}^{1,2}$ \\ Tianxin Qiu ${ }^{1,2}$ \\ Zhenguang Liu \\ Yuanliang $\mathrm{Hu}^{1,2}$ \\ jiaguo Liu $\mathbb{B}^{1,2}$ \\ Deyun Wang $\mathbb{B D}^{1,2}$ \\ 'Institute of Traditional Chinese \\ Veterinary Medicine, College of \\ Veterinary Medicine, Nanjing, People's \\ Republic of China; ${ }^{2}$ MOE Joint \\ International Research Laboratory of \\ Animal Health and Food Safety, College \\ of Veterinary Medicine, Nanjing \\ Agricultural University, Nanjing 210095 , \\ People's Republic of China
}

Background: Poly(lactic-co-glycolic acid) (PLGA) has been extensively applied for sustained drug delivery and vaccine delivery system. However, vaccines delivered by PLGA nanoparticles alone could not effectively activate antigen-presenting cells (APCs) to induce strong immune responses.

Purpose: The aim of the present study was to design polyethylenimine (PEI)-modified Chinese yam polysaccharide (CYP)-encapsulated PLGA nanoparticles (CYPP-PEI) as a vaccine delivery system and evaluate the adjuvant activities in vitro and in vivo.

Materials and Methods: Cationic-modified nanoparticles exhibited high antigen absorption and could be efficiently taken by APCs to enhance the immune responses. Therefore, PEI-modified CYP-encapsulated PLGA nanoparticles (CYPP-PEI) were prepared. The storage stability and effective adsorption capacity for porcine circovirus-2 (PCV-2) antigen of these antigen-absorbed nanoparticles were measured for one month. Furthermore, the adjuvant activity of CYPP-PEI nanoparticles was evaluated on macrophages in vitro and through immune responses triggered by PCV-2 antigen in vivo.

Results: The PCV-2 absorbed CYPP-PEI nanoparticles showed excellent storage stability and high absorption efficiency of PCV-2 antigen. In vitro, CYPP-PEI nanoparticles promoted antigen uptake, enhanced surface molecular expressions of CD80 and CD86, and improved cytokine secretion of TNF- $\alpha$, IFN- $\gamma$, and IL-12p70 in macrophages. After immunization with CYPP-PEI/PCV-2 formulation in mice, the expressions of surface activation markers on dendritic cells which located in draining lymph nodes were increased, such as MHCI, MHCII, and CD80. In addition, CYPP-PEI nanoparticles induced dramatically high PCV2-specific IgG levels which could last for a long time and stimulated the secretion of subtype antibodies and cytokines. The results showed that CYPP-PEI could induce Th1/Th2 mixed but Th1-biased type immune responses.

Conclusion: Polyethylenimine-modified Chinese yam polysaccharide-encapsulated PLGA nanoparticle was a potential vaccine delivery system to trigger strong and persistent immune responses.

Keywords: Chinese yam polysaccharide, polyethylenimine, poly(lactic-co-glycolic acid), nanoparticles, macrophages, immune response

\section{Introduction}

Tel +86-25-84395203

Fax +86-25-84398669

Email dywang@njau.edu.cn
Chinese yam polysaccharide (CYP), as one of the important active ingredients of Chinese yam, is comprised of $\beta$-1,3-glucose, $\alpha$-1-galactose, $\alpha$-1,6-galactose with 
a molecular weight of $1.66 \mathrm{kDa} .^{1}$ Diverse biological activities of Chinese yam polysaccharide have been demonstrated such as immunomodulatory, ${ }^{2,3}$ antioxidation, ${ }^{4,5}$ antitumor. $^{6,7}$ Recently, the immunomodulatory activity of yam polysaccharide attracted increasing attention. ${ }^{8,9}$

The drug delivery system was developed to sustain drug release and facilitate the drug bioavailability. ${ }^{10}$ Poly(lactic-co -glycolic acid) (PLGA), a popular nanomaterial with favorable degradation and hydrophilicity, has been broadly applied for sustainable drug delivery and vaccine delivery system. ${ }^{11-13}$ However, PLGA nanoparticles alone as vaccines delivery system could not effectively activate APCs and induce strong immune responses. It is necessary to use PLGA nanoparticles together with immunomodulatory adjuvants. ${ }^{14}$ Numerous researches have found that PLGA nanoparticles with positive surface charge had significant potential as an antigen delivery system. ${ }^{15-17}$ In particular, cationic-modified nanoparticles exhibited higher antigen absorption and could be efficiently taken by APCs compared with neutral charged nanoparticles. ${ }^{17,18}$ Porcine circovirus type 2 (PCV-2), the virus caused a kind of epidemic viral infectious disease in the porcine population, could result in diverse clinical syndromes, such as postweaning multisystemic wasting syndrome (PMWS), porcine dermatitis and nephropathy syndrome (PDNS), and respiratory disease. ${ }^{19}$ Although vaccination is a common method to control PCV-2 infection, for now subunit vaccines and inactivated vaccines cannot against pathogenic infections effectively. ${ }^{20,21}$ Therefore, we hypothesized that cationic-modified CYPencapsulated PLGA nanoparticle was a high potential vaccine adjuvant to enhance immune efficacy of PCV-2 vaccine.

Macrophages are important phagocytes and contribute to the innate immune responses and the adaptive immune responses, which could do a critical job in resisting pathogenic infections. ${ }^{22,23}$ Furthermore, the activation of macrophages could upregulate the level of costimulatory molecule expression such as CD80, CD86, CD40, MHC-II and the cytokines like IL10, IL-12, TNF- $\alpha$ and IFN- $\gamma .{ }^{22,24}$ Previous studies have demonstrated that nanoparticle-based vaccine delivery system improved the antigen uptake capacity of APCs (Dendritic cells, macrophages, etc.), which could contribute to enhancing immune responses. ${ }^{25,26}$

The purpose of this study was to design and prepare the polyethylenimine (PEI)-modified CYP-encapsulated PLGA nanoparticles (CYPP-PEI) and determine whether cationic-modified CYPP-PEI nanoparticles could enhance the effect of macrophages in vitro and facilitate the immune responses after immunization with PCV-2 antigen-absorbed nanoparticles in vivo.

\section{Materials and Methods Materials}

PLGA (lactic:glycolic ratio 75:25, MW: $15 \mathrm{kDa}$ ) was bought from Jinan Daigang Biomaterial Co. Ltd (Shandong, China). Chinese yam polysaccharide (CYP), Sephadex G-50, and Pluronic F68 (F68) were purchased from Yuanye Biomaterial Co. Ltd (Shanghai, China). 3-(4,5-dimethylthiazol-2-yl)-2,5-diphenyltetrazolium bromide (MTT) and Polyethylenimine (PEI) (MW: $25 \mathrm{kDa}$ ) were obtained from Sigma-Aldrich (USA). Inactivated PCV-2 antigen was supplied by Jiangsu Academy of Agricultural Science. BCA Protein Assay Kit and OVA-FITC were bought from Solarbio Science Technology Co. Ltd. (Beijing, China). The ELISA Kit to detect the antibody against Porcine Circovirus Type 2 was purchased from KeQian Biology Co., Ltd (Wuhan, China), Goat Anti-Mouse IgG/HRP was purchased from Biosynthesis Biotechnology Co., Ltd (Beijing, China).

\section{Preparation of Nanoparticles}

PEI-modified CYP-encapsulated PLGA nanoparticles (CYPP-PEI) were prepared by water-oil-water $(\mathrm{W} / \mathrm{O} / \mathrm{W})$ double emulsion solvent evaporation method as described previously. ${ }^{27-29}$ Briefly, the CYP aqueous solution $(20 \mathrm{mg}$ / $\mathrm{mL})$ was mixed with PLGA acetone solution $(20 \mathrm{mg} / \mathrm{mL})$ with the volume ratio of 1:9. After sonicating for $2 \mathrm{~min}$, the mixture was dispersed into $0.7 \%$ F68 aqueous with the volume ratio of 1:10 and sonicated for $3 \mathrm{~min}$. The emulsion was stirred for $3 \mathrm{~h}$ to obtain the stable double emulsion. To be further modified with PEI, different weight of PEI was added into the stable double suspension to yield various PLGA/PEI ratios and applied to achieve enough modification with magnetic stirring for $3 \mathrm{~h}$ at room temperature (The PLGA:PEI weight ratio of 10:1, 20:1, 30:1 was, respectively, expressed as CYPP-PEI 1, CYPP-PEI 2, CYPP-PEI 3). The nanoparticles were centrifuged (3000 rpm, $10 \mathrm{~min}$ ) and the supernatant was obtained. The preparation procedures of CYP-PLGA nanoparticles (CYPP) were the same as CYPP-PEI nanoparticles without PEI, and the Blank PLGA (BP) nanoparticles were synthesized without CYP and PEI.

To obtain the PCV-2 antigen-adsorbed nanoparticles, the nanoparticles were synthesized in a similar approach as mentioned above and then different formulations were 
vortexed with an equal volume of inactivated PCV-2 antigen separately for $3 \mathrm{~min}$ and then stored at $4^{\circ} \mathrm{C}$ for $12 \mathrm{~h}$. Transmission electron microscopy (TEM) was applied to detect the morphology of PCV-2 antigen-absorbed nanoparticles.

\section{Characterization of Nanoparticles}

The average size, zeta potential, and polydispersity index (PDI) were examined by Zetasizer Nano Instruments (Hydro2000Mu, MAL1009117, UK). The surface morphology of nanoparticles was photographed by TEM (Tecnai 12, Philips, Holland).

The CYP encapsulation efficiency of CYPP nanoparticles and CYPP-PEI nanoparticles was determined using modified microcolumn centrifugation method and phenol-sulfuric acid method. ${ }^{30,31}$ Briefly, Sephadex G-50 was dispersed in deionized water overnight and centrifuged (1500 rpm, $5 \mathrm{~min}$ ) to remove excess fluid before the Sephadex-50 microcolumns were fabricated. Subsequently, nanoparticles were added into the microcolumn and centrifuged (2000 rpm, $10 \mathrm{~min}$ ), after which free CYP could separate from the nanoparticles.

The encapsulation efficiency (EE) of CYP was accessed by the phenol-sulfuric acid method and calculated as follows:

$$
E E(\%)=C e / C t \times 100
$$

where $\mathrm{EE}$ is the encapsulation efficiency, $\mathrm{Ct}$ is the total amount of CYP, and Ce is the encapsulated amount of CYP.

\section{Stability of PCV-2 Antigen-Absorbed Nanoparticles}

The CYPP-PEI nanoparticles and PCV-2 absorbed nanoparticles were quiescently stored at $4{ }^{\circ} \mathrm{C}$. In order to test storage stability, the average size, zeta potential, and PDI were examined on day $7,14,21$, and 28 , respectively.

The stability of PCV-2 adsorption efficiency was measured by a BCA kit at the same intervals over 28 days. In order to measure the adsorption efficiency of PCV-2, the prepared PCV-2-adsorbed nanoparticles were ultracentrifuged at $4^{\circ} \mathrm{C}, 12,000 \mathrm{rpm}$ for $30 \mathrm{~min}$. Then, the content of free PCV-2 antigen in the supernatant was measured and the $\mathrm{PCV}-2$ antigen adsorption rate (AR) was calculated as follows:

$$
E E(\%)=C e / C t \times 100
$$

where AR is the PCV-2 adsorption rate, $\mathrm{Ct}$ is the total content of antigen, and Cs is the content of antigen in the supernatant.

\section{Cytotoxicity Assessment}

Macrophages were harvested from the abdominal cavities of ICR mice as previous description. ${ }^{30}$ MTT method ${ }^{32}$ was used to analyze the cytotoxicity of CYP, CYPP, and CYPP-PEI nanoparticles on the macrophages. Cells were incubated with nanoparticles at the concentration of $0.785 \sim 200 \mu \mathrm{g} / \mathrm{mL}$ for $48 \mathrm{~h}$ and measured at $570 \mathrm{~nm}$ by the microplate reader (Thermo, USA).

\section{Analysis of Antigen Uptake by Macrophages}

The effect of antigen uptake was examined using OVA-FITC -loaded nanoparticles on macrophages by flow cytometry. The CYP, BP, CYPP, and CYPP-PEI nanoparticles were mixed with $10 \mu \mathrm{L}$ OVA-FITC for $1 \mathrm{~h}$ at room temperature to obtain the OVA-FITC-adsorbed nanoparticles. Macrophages were separately co-cultured with different prepared nanoparticles for $12 \mathrm{~h}$ and centrifuged, washed twice with PBS. The intensity of antigen uptake was assessed by flow cytometry (Accuri C6 flow cytometer BD Biosciences). Besides, macrophages were added into Petri dishes and incubated with OVA-FITC-adsorbed nanoparticles for $12 \mathrm{~h}$. The cells were washed and mixed with $4 \%$ formaldehyde for 10 min after incubating. DAPI was used to stain cell nucleus for $5 \mathrm{~min}$ and the uptake of nanoparticles was observed by confocal microscopy (NikonA1). ${ }^{33}$

\section{The Activation of Macrophages}

The macrophages were incubated separately with CYP, BP, CYPP, and CYPP-PEI nanoparticles (12.5 $\mu \mathrm{g} / \mathrm{mL})$ for $48 \mathrm{~h}$. After being washed and purified, the macrophages were stained with CD80 (B7-1) and CD86 (B7-2) antibodies (eBioscience, USA) for $1 \mathrm{~h}$ at $4^{\circ} \mathrm{C}$ in the dark. Finally, macrophages were washed with PBS and analyzed by flow cytometry.

In order to measure the levels of TNF- $\alpha$, IFN- $\gamma$, and IL-12p70, the culture was collected after $48 \mathrm{~h}$ and then analyzed by cytokine-specific ELISA Kits (Multi Sciences) according to the manufacturer instructions.

\section{Immunizations and Samples Collection}

Seven-week ICR mice were purchased from Comparative Medicine Centre of Yangzhou University. The standard 
pelleted rodent chow and water were supplemented. All animal experiments were performed as the guideline of Nanjing Agricultural University IACUC and the protocol was approved by the Institutional Animal Care and Use Committee (IACUC) (No. 2011BAD34B02).

Seven groups of mice were injected subcutaneously with $200 \mu 1$ of different formulations, respectively: CYPP-PEI

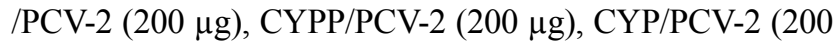
$\mu \mathrm{g}$ ), BP/PCV-2, ISA206/PCV-2, PCV-2, and PBS. (ISA-206/ $\mathrm{PCV}-2$ was used as the positive control, PBS was used as the negative control). Booster immunization was performed on day 14 after primary immunization. Four mice were selected randomly from each group to collect blood samples on days $21,28,35$, and 42 after primary immunization, the serum was then collected and stored at $-80^{\circ} \mathrm{C}$.

\section{Effect of Nanoparticles on Dendritic Cell in Draining Lymph Nodes}

Four mice were randomly selected from each group at 24 $\mathrm{h}$ after primary immunization. The lymph nodes were collected from them and homogenized to obtain the single cell suspension. CD11c, MHCI (H-2kd/H-2Dd), MHCII (I-A/I-E), and CD80 (B7-1) antibodies (eBioscience, USA) were used to stain DCs at $4^{\circ} \mathrm{C}$ for $1 \mathrm{~h}$. Finally, cells were washed and measured by flow cytometry.

\section{ELISA Analysis of PCV-2-Specific IgG and Isotypes}

In order to estimate the level of PCV-2-specific IgG, the serum samples were measured by modified ELISA Kits. The plates were washed twice with diluted wash buffer; then $100 \mu \mathrm{l}$ of diluted serum samples (1:2560) were added into plates and incubated at $37^{\circ} \mathrm{C}$ for $30 \mathrm{~min}$. After being washed three times, $100 \mu \mathrm{l}$ of HRP-conjugated goat antibodies against $\operatorname{IgG}$ were added and incubated at $37^{\circ} \mathrm{C}$ for 30 min. After being washed five times, plates were incubated with $100 \mu \mathrm{l}$ of the TMB at room temperature for $10 \mathrm{~min}$ avoid light and the stop reagent was added. The absorbance was measured at $630 \mathrm{~nm}$ by the microplate reader.

The serum PCV-2-specific IgG1, IgG2a, IgG2b antibody levels were examined by ELISA Kits (Multi Sciences) on days $21,28,35$, and 42 after primary immunization according to the manufacturer protocol.

\section{Evaluation of Cytokines Levels by ELISA}

The cytokines concentrations of TNF- $\alpha$, IFN- $\gamma$, IL-4, and IL-6 in serum were examined by ELISA Kits (Multi
Sciences) on days 21 and 35 after primary immunization according to the manufacturer protocol.

\section{Statistical Analysis}

All values are expressed as mean \pm standard error of the mean (SEM). The statistical significance was accessed using Duncan's multiple range test. A probability value (P) less than 0.05 was considered statistically significant.

\section{Results \\ Synthesis and Characterization of Polyethylenimine Modificated Nanoparticles}

As shown in Figure 1A, Polyethylenimine (PEI)-modified Chinese yam polysaccharide (CYP) PLGA nanoparticles (CYPP-PEI) were prepared by double emulsion (W/O/W) solvent evaporation method. Cationic surface of PLGA nanoparticles was formed using PEI, which facilitated the adsorption of negative PCV-2 antigen. Size, zeta potential, and PDI were measured to optimize the ratio of PLGA: PEI weight in nanoparticles preparation. As shown in Figure 1B, the size of CYPP-PEI1, CYPP-PEI2, and CYPP-PEEI3 nanoparticles was $246.2 \mathrm{~nm}, 236.6 \mathrm{~nm}$, and $256.9 \mathrm{~nm}$, respectively, which were larger than CYPP nanoparticles $(225.0 \mathrm{~nm})$. In Figure 1C the CYPP nanoparticles showed negative surface charges $(-20.7 \mathrm{mV})$, while CYPP-PEI1, CYPP-PEI2, and CYPP-PEI3 nanoparticles showed positive charges $(+42.1 \mathrm{mV},+41.5 \mathrm{mV}$, and $+32 \mathrm{mV}$, respectively). These results suggested that when the ratio of PLGA: PEI weight was 10:1, 20:1 (CYPPPEI1 and CYPP-PEI2), CYPP-PEI could have higher surface positive charges compared to the ratio was 30:1 (CYPP-PEI3). PDI values of CYPP-PEI1 and CYPPPEI2 were similar to CYPP nanoparticles, but the PDI of CYPP-PEI3 was higher than other groups (0.319). The encapsulation efficiency of CYPP, CYPP-PEI, CYPPPEI2, and CYPP-PEI3 was $64.8 \pm 4.2 \%, 49.7 \pm 3.1 \%, 59$ $\pm 2.3 \%$, and $62.7 \pm 3.4 \%$, respectively (Figure 1D), indicated that the encapsulation efficiency of CYPP-PEI1 nanoparticles was obviously decreased compared with CYPP nanoparticles. Therefore, 20:1 (CYPP-PEI2) was the optimal ratio of PLGA: PEI weight to prepare PEImodified nanoparticles, which showed average size of $236.6 \mathrm{~nm}$, positive charges of $+41.5 \mathrm{mV}$, PDI value of 0.149 and high encapsulation efficiencies (EE) of 59 $\pm 2.3 \%$. Representative TEM images of the CYPP nanoparticles and CYPP-PEI nanoparticles (Figure 1E) have 
CYPP-PEI nanoparticles

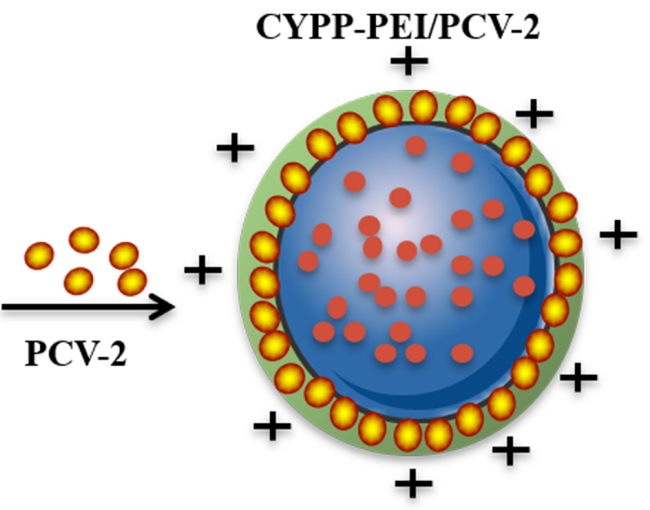

A

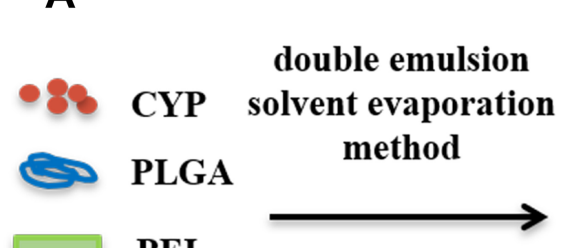

PEI

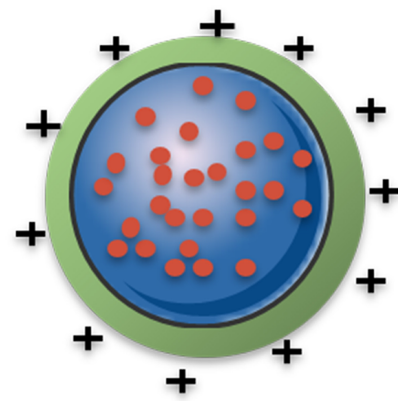

B
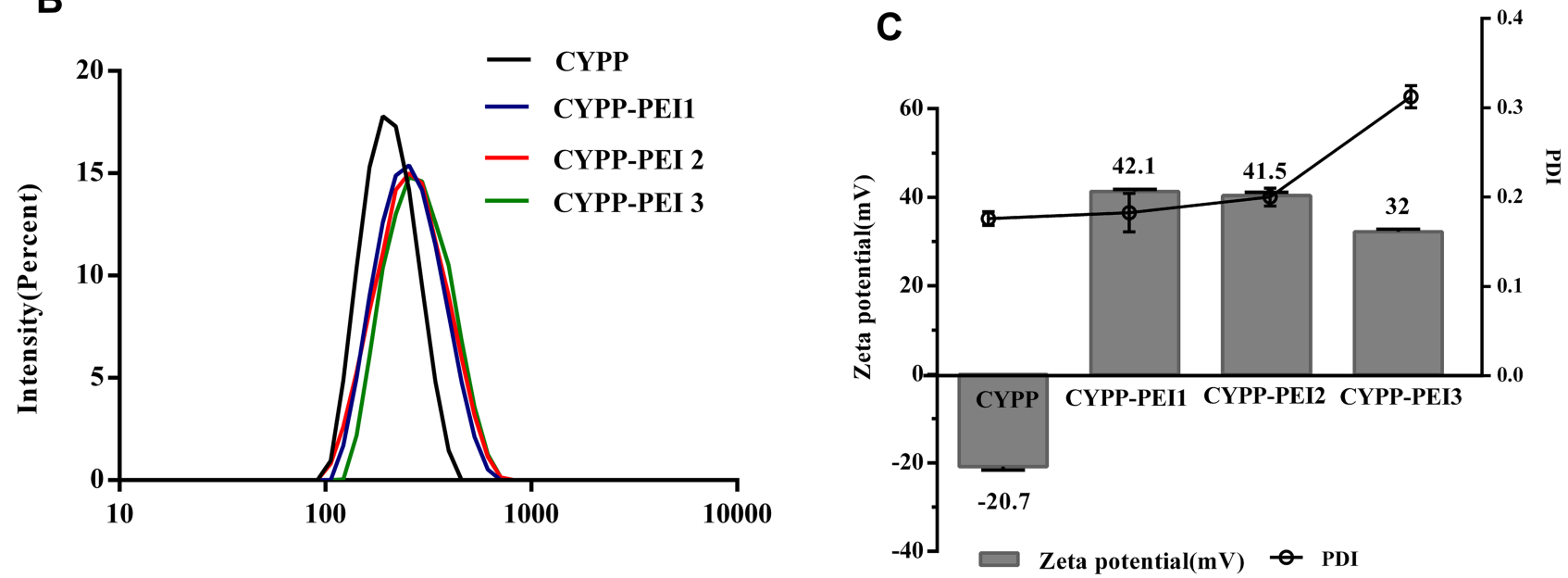

Size (d.nm)

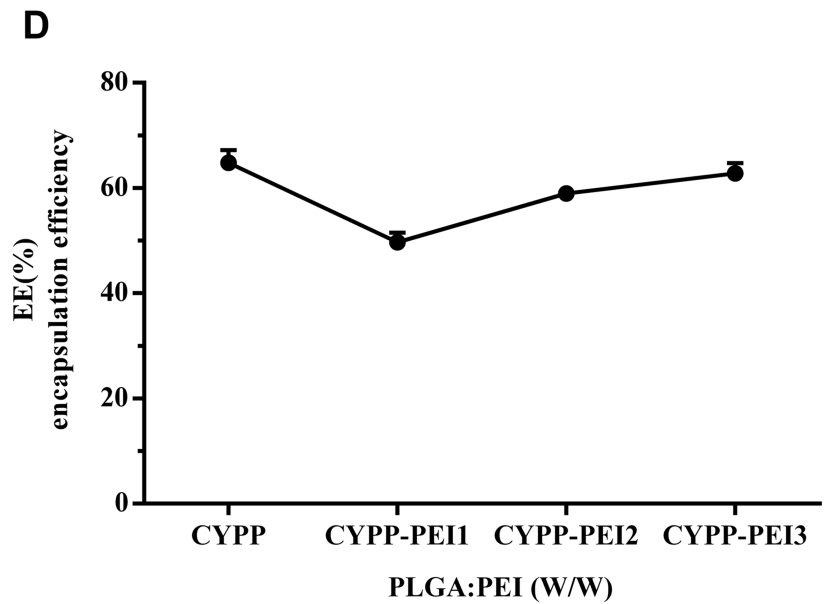

E
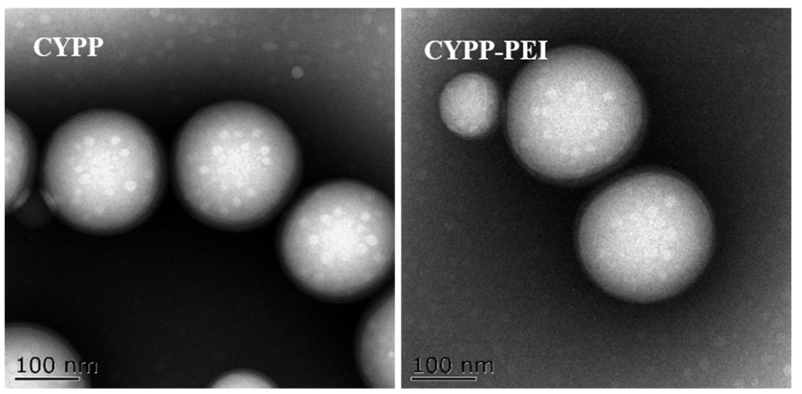

Figure I Preparation and characterization of nanoparticles. (A) Schematic illustration of CYPP-PEI nanoparticles and PCV-2 absorbed nanoparticles (CYPP-PEI/PCV-2). (B) size intensity, (C) zeta potential and Poly dispersity index (PDI) (D) encapsulation efficiency (EE\%) of CYPP nanoparticles and CYPP-PEI nanoparticles (Different PLGA: PEI weight ratio of 10: I, 20: I, 30: I were respectively expressed as CYPP-PEI I, CYPP-PEI 2, CYPP-PEI 3.). Typical TEM images of (E) CYPP nanoparticles and CYPP-PEI nanoparticles. Data were presented as the mean $\pm \operatorname{SEM}(n=3)$. 
shown that surface morphologies of CYPP-PEI nanoparticles were similar to CYPP nanoparticles while the sizes of CYPP-PEI nanoparticles were bigger than CYPP nanoparticles. These results indicated that PEI surface modification of nanoparticles had no influence on the morphology of nanoparticles.

\section{The Stability and Morphology of Nanoparticles and PCV-2 \\ Antigen-Absorbed Nanoparticles}

To estimate the storage stability of nanoparticles and PCV-2 antigen-absorbed nanoparticles, size, zeta potential, and PDI value were measured at $4^{\circ} \mathrm{C}$ for 1 month. In Figure 2A, the average size was increased slightly from $176.2 \mathrm{~nm}$ to $239.16 \mathrm{~nm}$ for BP nanoparticles, $239.1 \mathrm{~nm}$ to $239.2 \mathrm{~nm}$ for CYPP nanoparticles and $246.2 \mathrm{~nm}$ to 281.9 $\mathrm{nm}$ for CYPP-PEI nanoparticles. In Figure 2B, the zeta potential of BP and CYPP nanoparticles showed negative surface charge approximately $-20 \mathrm{mV}$, while CYPP-PEI showed positive charge from $53.6 \mathrm{mV}$ to $45.0 \mathrm{mV}$. In Figure 2C, the PDI value of BP, CYPP, and CYPP-PEI nanoparticles were less than 0.3 . The average size, zeta potential, and PDI value of BP, CYPP, CYPP-PEI nanoparticles showed no significant changes and deviations, indicating that these nanoparticles showed high stability within a month. In Figure 2A, the average size of CYPPPEI/PCV-2 nanoparticles increased slightly from 409.2 $\mathrm{nm}$ to $449.1 \mathrm{~nm}$, the average size of CYPP/PCV-2 nanoparticles increased from $245.7 \mathrm{~nm}$ to $260.1 \mathrm{~nm}$ and the average size of $\mathrm{BP} / \mathrm{PCV}-2$ nanoparticles changed from $213.7 \mathrm{~nm}$ to $209.5 \mathrm{~nm}$. Compared with $\mathrm{BP} / \mathrm{PCV}-2$ and CYPP/PCV-2 nanoparticles, the average size of CYPPPEI showed dramatically increased, indicating that more PCV-2 antigen absorbed on the surface of CYPP-PEI nanoparticles. In Figure $2 \mathrm{~B}$ and $\mathrm{C}$, there were no dramatical changes in zeta potential and PDI value of BP/PCV2, CYPP/PCV-2, and CYPP-PEI/PCV-2 nanoparticles. The zeta potential was nearly neutral, while the PDI value was all less than 0.3 , indicating that PCV-2 antigenabsorbed nanoparticles maintained uniformity within a month. These results suggested that nanoparticles and PCV-2 antigen-absorbed nanoparticles were stable at $4{ }^{\circ} \mathrm{C}$ for a month.

The PCV-2 adsorption efficiency of nanoparticles was measured by BCA protein assay kit. As shown in Figure 2D, the absorption efficiency ranged from $30.72 \%$ to $21.26 \%$ for CYPP-PEI/PCV-2 nanoparticles, $13.95 \%$ to $9.81 \%$ for
CYPP/PCV-2 nanoparticles, $11.85 \%$ to $7.85 \%$ for BP/PCV2 nanoparticles, respectively. The absorption efficiency of CYPP-PEI/PCV-2 was higher than CYPP/PCV-2 and BP/ PCV-2 nanoparticles within 1 month, which revealed that the cationic charge of CYPP-PEI nanoparticles enhanced the absorption efficiency of PLGA nanoparticles to PCV-2 antigens.

In Figure 2E, typical surface morphologies of the nanoparticles were observed by TEM, all nanoparticles had spherical structure with smooth surface. However, the size of CYPP-PEI/PCV-2 nanoparticles was larger than other nanoparticles, which indicated that PCV-2 antigen successfully absorbed on the surface of the CYPP-PEI nanoparticles.

\section{Effect of Nanoparticles on the Viability of Macrophages}

To access the effect of CYPP-PEI on macrophages, the viabilities of macrophages were measured by MTT assay. As shown in Figure 3, it was found that the macrophages retained more than $90 \%$ of viability after being treated with CYP, BP, and CYPP nanoparticles at the final concentrations ranging from $0.781 \mu \mathrm{g} / \mathrm{mL}$ to $200 \mu \mathrm{g} / \mathrm{mL}$. When the total concentration of CYPP-PEI nanoparticles was lower than $25 \mu \mathrm{g} / \mathrm{mL}$, the viability of cells was more than $90 \%$. In contrast, CYPP-PEI nanoparticles showed obvious cytotoxicity to macrophages at the concentration of $50 \mu \mathrm{g} / \mathrm{mL} \sim 200 \mu \mathrm{g} / \mathrm{mL}$. These data suggested that the cytotoxicity effect of CYPP-PEI nanoparticles may be caused by the PEI polymer. After all, PEI polymers have been widely used in vaccines, ${ }^{18}$ cancer treatment, ${ }^{15}$ and gene delivery, ${ }^{34}$ which indicated that the total concentration of CYPP-PEI nanoparticles was lower than $25 \mu \mathrm{g} / \mathrm{mL}$ may be safe for vaccination.

\section{Cellular Uptake of Nanoparticles}

The cellular uptake capacity of macrophages towards OVA-FITC loaded CYPP-PEI nanoparticles or OVAFITC was measured by flow cytometry and CLSM.

The proportion of positive fluorescence cells in CYPPPEI group was dramatically higher than which in CYPP, $\mathrm{BP}, \mathrm{CYP}$, and control groups $(\mathrm{P}<0.05)$ (Figure 4A and $\mathrm{B}$ ), indicating that CYPP-PEI nanoparticles enhanced the antigen uptake of macrophages.

CLSM was further used to measure the uptake capacity. In Figure 4C, compared with CYPP, BP, CYP, and control group, CYPP-PEI nanoparticles group showed 

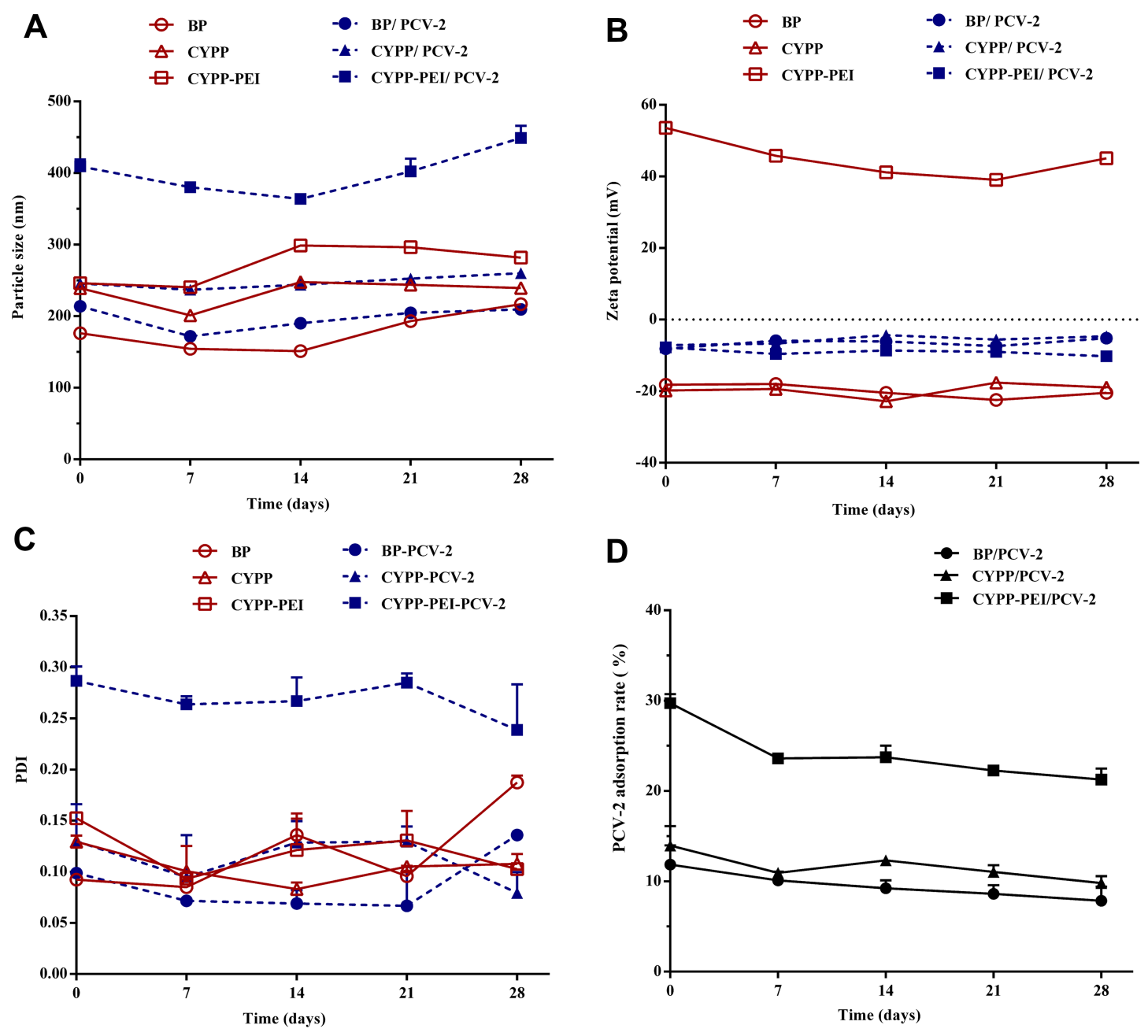

\section{$\mathbf{E}$}
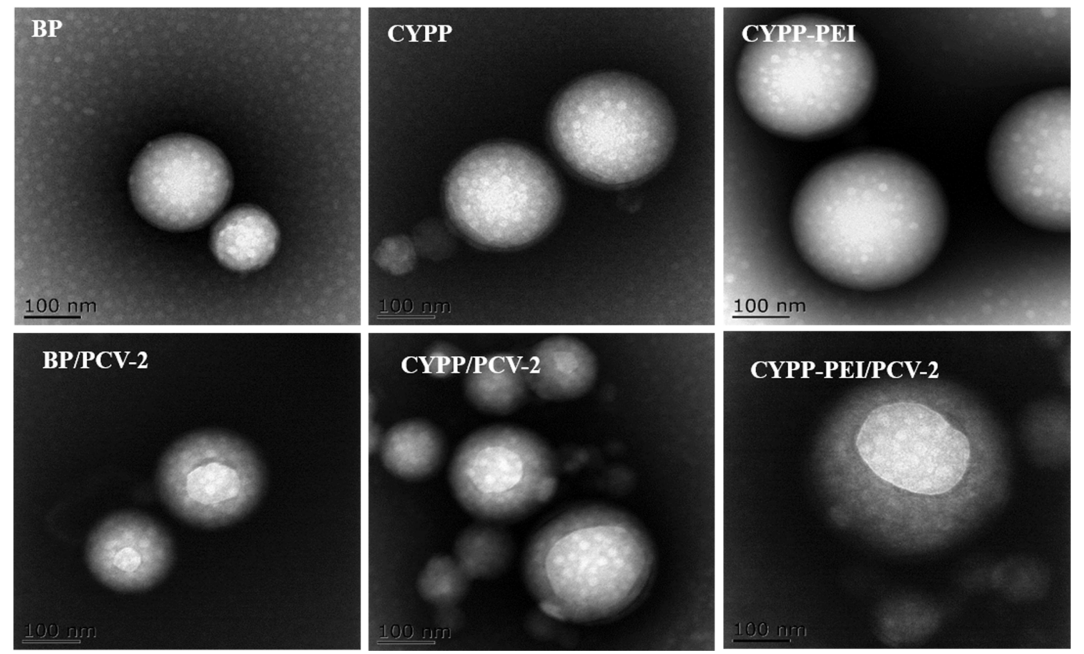

Figure 2 The Stability of (A) size intensity, (B) zeta potential, and (C) PDI of BP, CYPP, CYPP-PEI, BP/PCV-2, CYPP/PCV-2, and CYPP-PEI/PCV-2 stored at $4{ }^{\circ} \mathrm{C}$ for a month. (D) The PCV-2 antigen absorption efficiencies of BP/PCV-2, CYPP/PCV-2, and CYPP-PEI/PCV-2 stored at $4{ }^{\circ} \mathrm{C}$ for a month. (E) TEM images of BP, CYPP, CYPP-PEI nanoparticles, and PCV-2 antigen-absorbed nanoparticles: BP/PCV-2, CYPP/PCV-2, CYPP-PEI/PCV-2 nanoparticles. Results were expressed as mean \pm SEM $(n=3)$. 


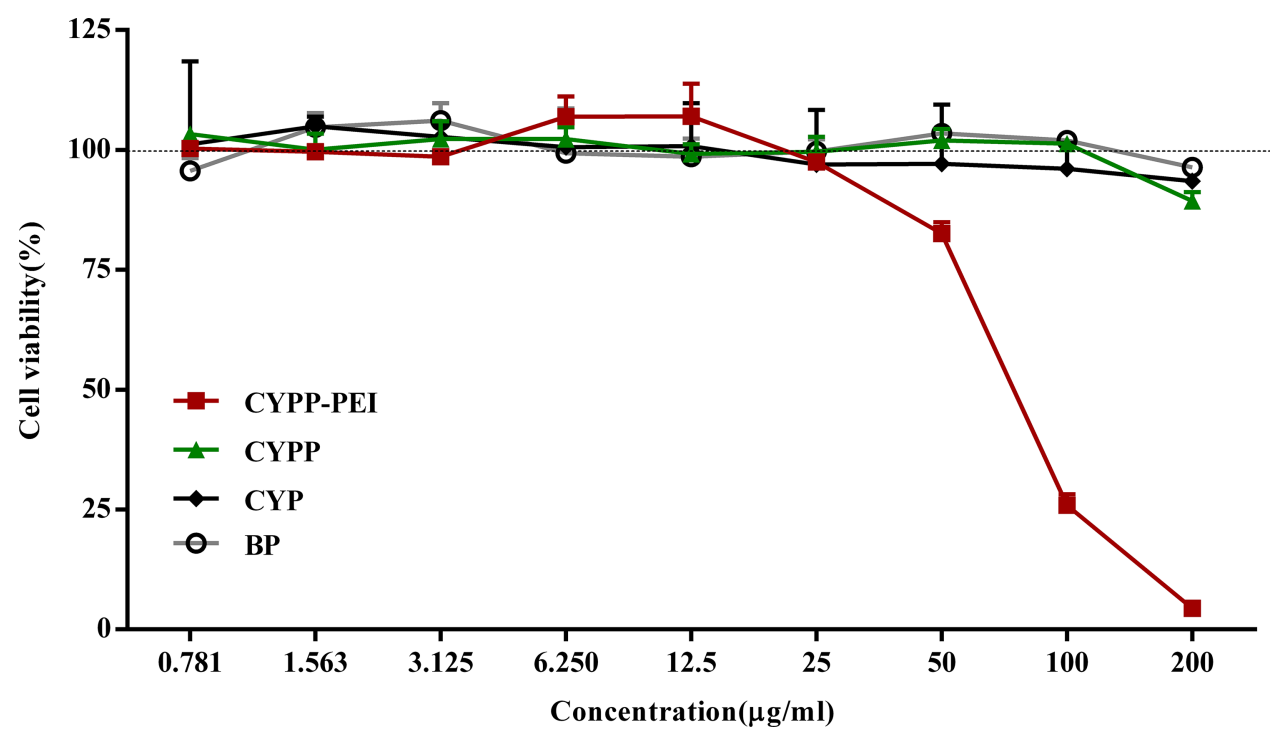

Figure 3 MTT assay results of macrophages after being incubated with different concentration of CYPP-PEI, CYPP, CYP, and BP nanoparticles for 48 h. Data are presented as the mean $\pm \operatorname{SEM}(n=4)$.

stronger green fluorescence in macrophages. These results could be well explained by the high efficiency ratio of FITC-OVA+ fluorescence cells treated with CYPP-PEI nanoparticles in flow cytometry plot, which revealed that the cationic CYPP-PEI obviously promoted the uptake capacity of macrophages.

\section{Efficient Activation of Macrophages with CYPP-PEI Nanoparticles}

Macrophages were plated in 24-well plates at a density of $5 \times 10^{5}$ cells/well and incubated with nanoparticles for 48 h. The expressions of surface molecular CD80 and CD86 were examined by flow cytometry. The quantitative analysis and flow cytometry plots are shown in Figure 5AC, macrophages treated with CYPP-PEI nanoparticles showed noticeable increases in CD80 and CD86 expression compared with CYPP, BP, CYP, and control groups $(\mathrm{P}<0.05)$. Moreover, the $\mathrm{CD} 80$ and $\mathrm{CD} 86$ expressions of CYPP nanoparticles were higher than BP, CYP, and control groups $(\mathrm{P}<0.05)$. These data demonstrated that both CYPP-PEI and CYPP nanoparticles could enhance activation of macrophages by stimulating the secretion of CD80 and CD86 surface molecular. Not only that, the activation ability of CYPP-PEI was stronger than CYPP nanoparticles. Subsequently, the cytokines secretions of IFN- $\gamma$, IL$12 \mathrm{p} 70$, and TNF- $\alpha$ were determined by ELISA. As shown in Figure 5DF, CYPP-PEI nanoparticles dramatically enhanced the secretions of IFN- $\gamma$, IL-12p70, and TNF- $\alpha$ compared to CYPP, BP, CYP, and control group $(\mathrm{P}<0.05)$.

\section{In vivo Study of Surface Activation Markers Expression on Dendritic Cells in Draining Lymph Nodes}

Delivering antigen to draining lymph nodes (DLNs) plays a significant role in initiating and adaptive immune response. Efficient activation of dendritic cells (DCs) in LN enhanced the immune response. ${ }^{35}$ To determine whether the CYPP-PEI nanoparticles could influence the activation of DCs in DLNs, the surface activation markers of MHCI, MHCII, and CD80 were measured by flow cytometry. The DLNs were collected from mice immunized with PCV-2 absorbed nanoparticles at $24 \mathrm{~h}$ after immunization, PBS was used as negative control. The percentage of $\mathrm{MHCI}+\mathrm{CD} 11 \mathrm{c}+$ (Figure $6 \mathrm{~A}$ and $\mathrm{D}$ ) and $\mathrm{CD} 80+\mathrm{CD} 11 \mathrm{c}+($ Figure $6 \mathrm{C}$ and $\mathrm{F}$ ) in CYPP-PEI/PCV-2 nanoparticles group showed a dramatic increase compared with other groups $(\mathrm{P}<0.05)$. Meanwhile, CYPP/PCV-2 and CYPP-PEI/PCV-2 nanoparticles groups were expressed higher level of $\mathrm{MHCII}+\mathrm{CD} 11 \mathrm{c}+$ than other groups $(\mathrm{P}<0.05)$ as shown in Figure 6B and $\mathrm{E}$. These data indicated that the positive surface charge of CYPP-PEI nanoparticles promoted the activation of DCs in DLNs.

\section{Serum Antibody Response After Vaccination in Mice}

To determine the effect of different nanoparticles on antibody responses, the PCV-2-specific IgG, IgG1, IgG2a, and IgG2b antibody levels were examined by ELISA. As shown in 


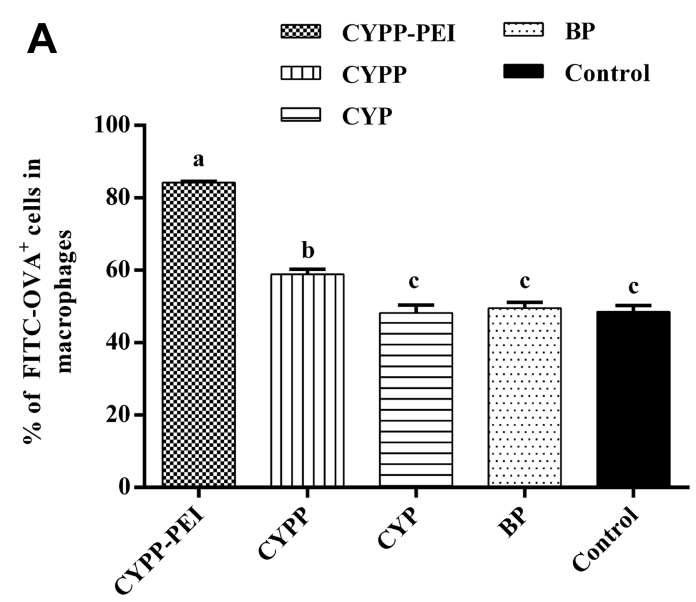

B
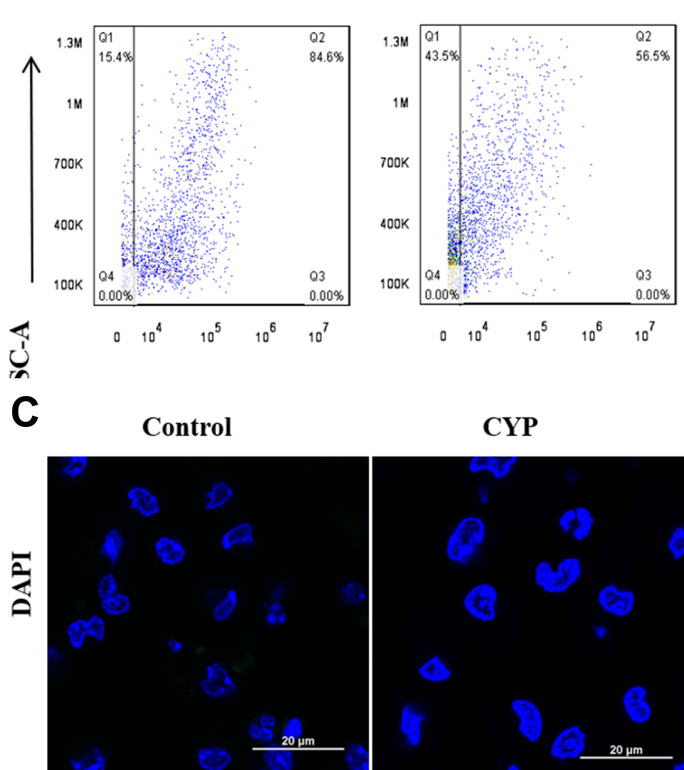

CYP
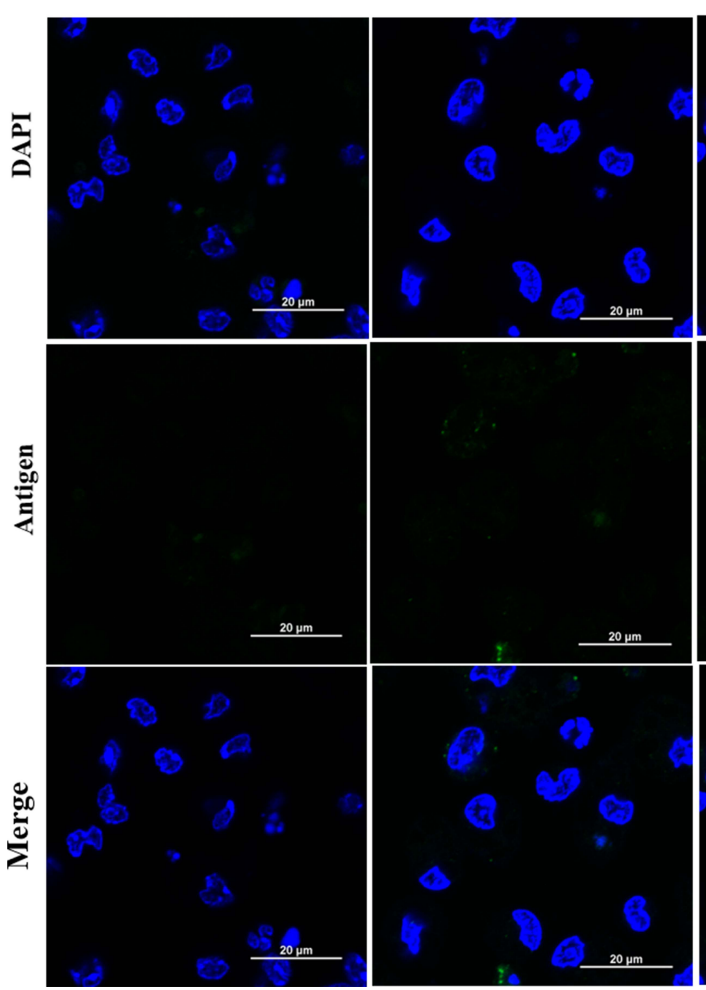
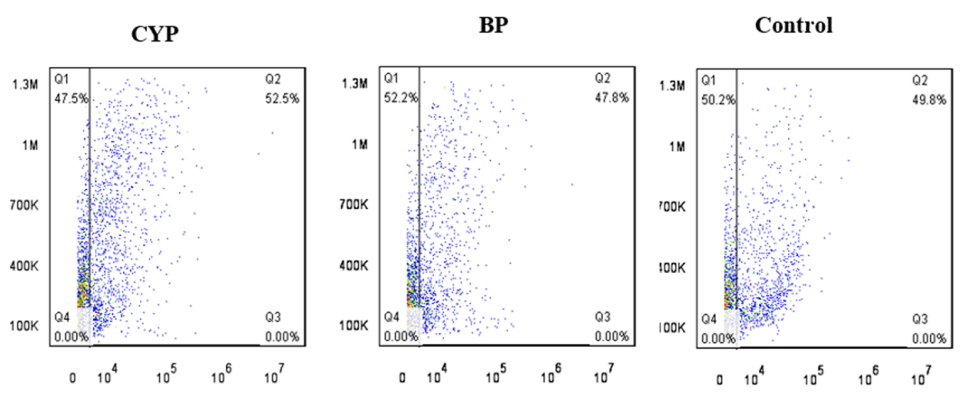

BP

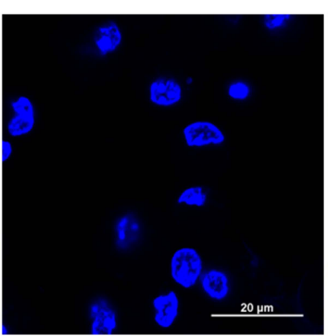

CYPP

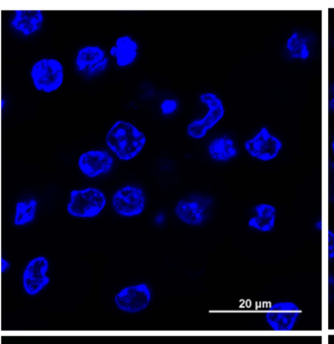

CYPP-PEI
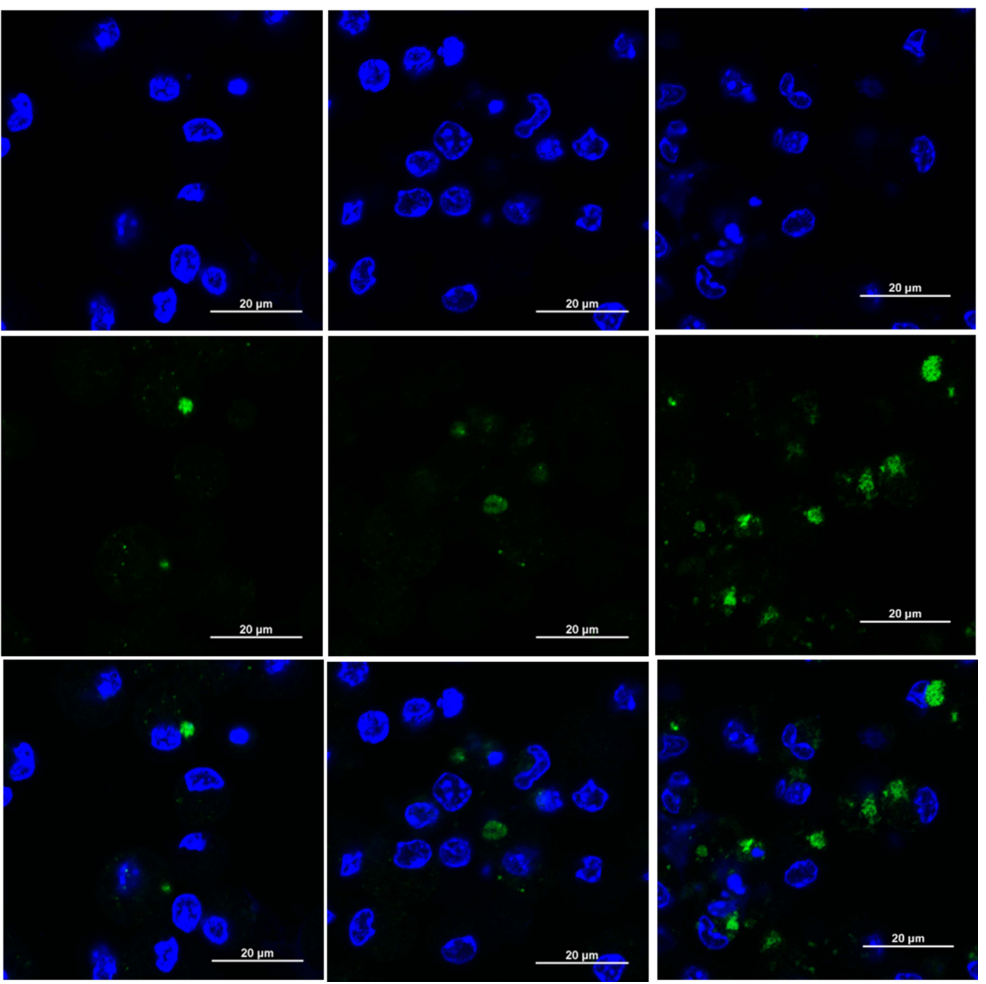

Figure 4 Antigen uptake in macrophages. Different nanoparticles CYPP-PEI, CYPP, CYP, and BP containing FITC-OVA were incubated with macrophages for I2 h. FITCOVA was used as control. (A) Analysis of FITC-OVA ${ }^{+}$antigen in macrophages by flow cytometry. (B) Representative flow cytometry plots of macrophages incubated with FITC-OVA. (C) Representative confocal laser scanning images of phagocytic macrophages. OVA was labeled by FITC (green) and nucleus were stained with DAPI (blue). Scale bar $=20 \mathrm{~nm}$. Results were expressed as means \pm SEM $(n=3)$. a-c Bars in the figure without the same superscripts differed significantly $(P<0.05)$. 

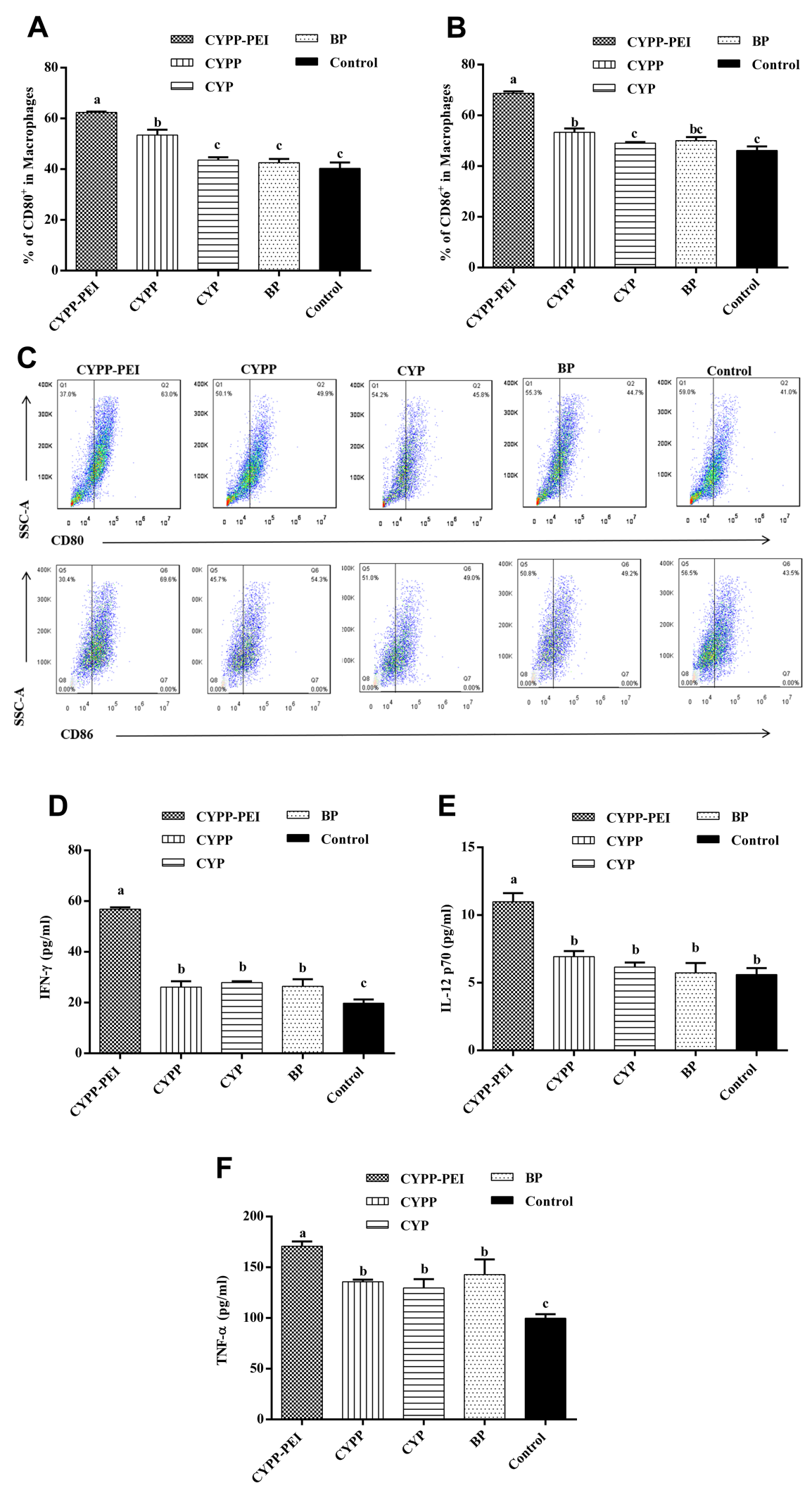

Figure 5 The activation effects of different nanoparticles to macrophages. Nanoparticles were incubated with mouse peritoneal macrophages for $48 \mathrm{~h}$ and the surface molecule expressions of (A) CD80, (B) CD86 in macrophages were analyzed by flow cytometry. (C) Representative flow cytometry plots of CD80 and CD86 surface molecule expression. Cytokine release (D) IFN- $\gamma,($ E) IL- I2P70, and (F) TNF- $\alpha$ were measured by ELISA kits. Results were expressed as means \pm SEM $(n=3)$. a-c Bars in the figure without the same superscripts differed significantly $(P<0.05)$. 

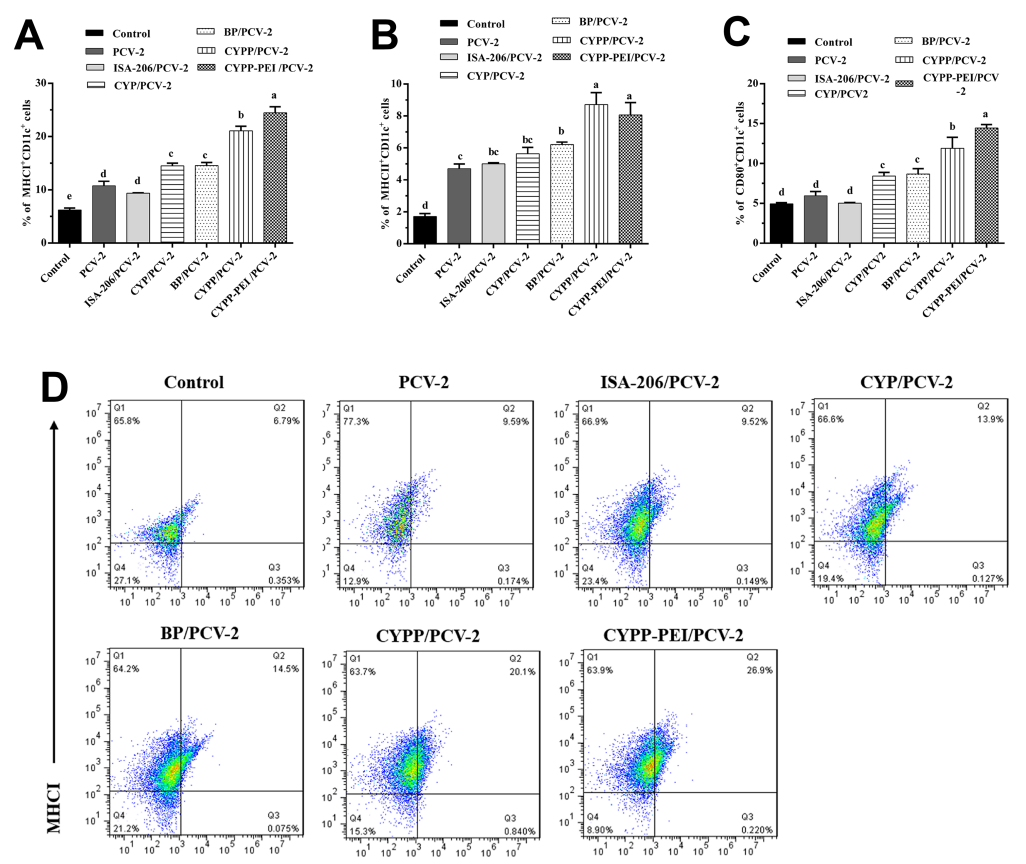

CD11c
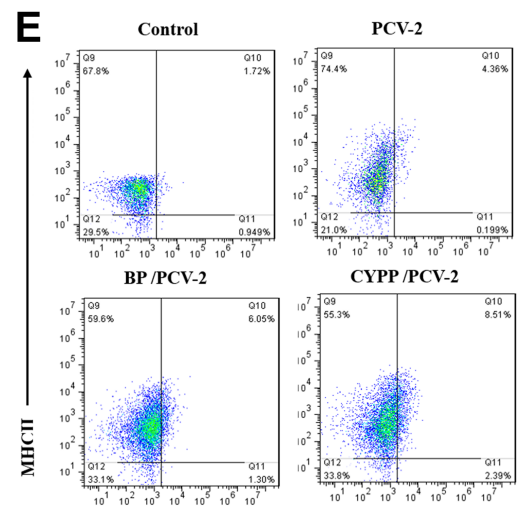

ISA-206/PCV-2

$$
\text { CD11c }
$$

$\mathbf{F}$
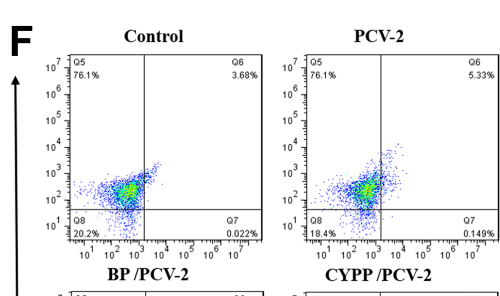

ISA-206/PCV-2
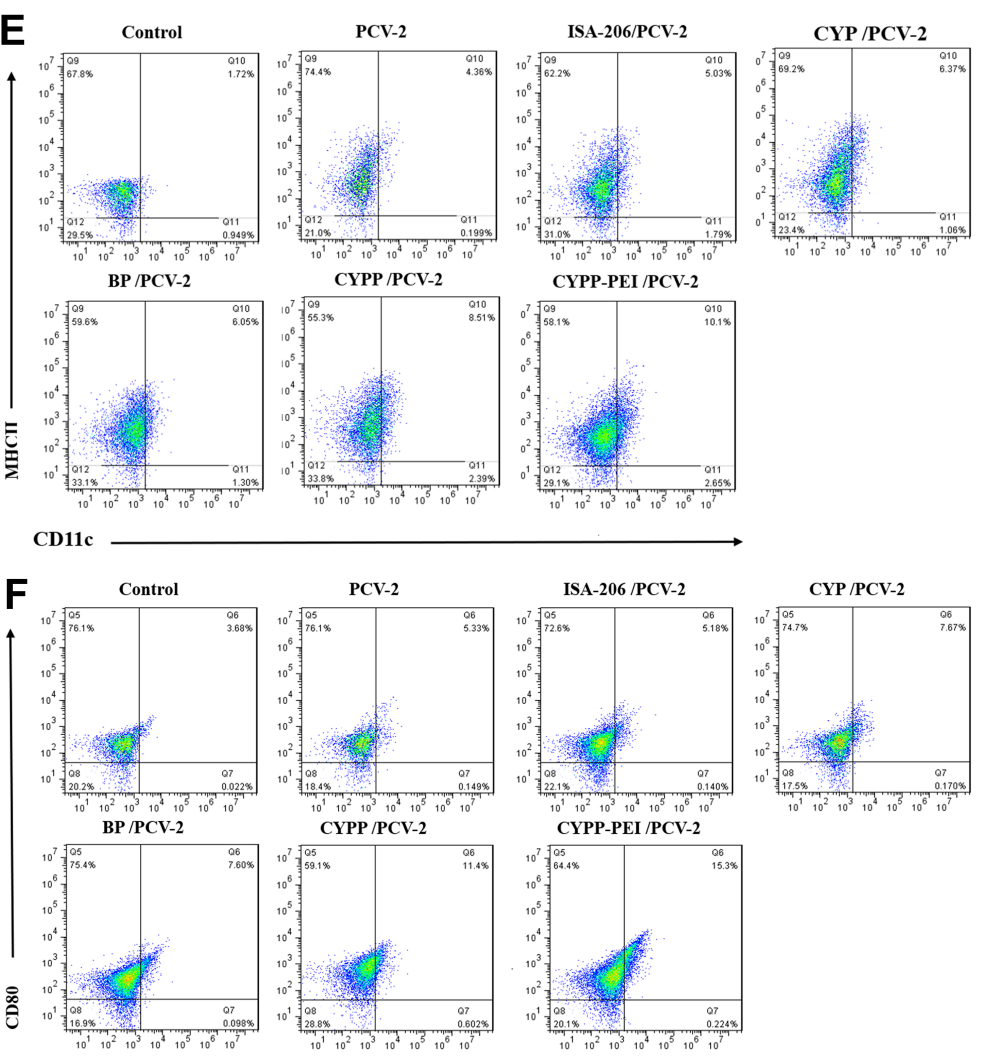

CD11c

Figure 6 The activation of dendritic cells in draining lymph nodes. Dendritic cells isolated from the draining lymph node of mice were collected at $24 \mathrm{~h}$ after immunization with different formulations of PBS, PCV-2, ISA-206/PCV-2, CYP/PCV-2, BP/PCV-2, CYPP/PCV-2, and CYPP-PEl/PCV-2. Analysis of surface molecule expression (A) MHCl, (B) $\mathrm{MHCll}$, and (C) CD80. Representative flow cytometry plots of (D) $\mathrm{MHCl},(\mathbf{E}) \mathrm{MHCll}$, and (F) CD80 surface activation markers expression in the lymph nodes. Results were expressed as mean $\pm \operatorname{SEM}(n=4)$. a-e Bars in the figure without the same superscripts differed significantly $(P<0.05)$. 
Figure 7A, seven groups of mice were subcutaneously immunized on days 0 and 14, the serum was collected and analyzed on days $21,28,35$, and 42 after primary immunization.

In Figure 7B, on days 28, 35, and 42, both CYPP-PEI/PCV2 and ISA-206/PCV-2 induced dramatically strong and persistent PCV-2-specific IgG expressions, which were significantly higher than the other groups $(\mathrm{P}<0.05)$. Moreover, CYPP/PCV2 nanoparticles were dramatically increased the PCV-2-specific IgG levels compared with CYP/PCV-2, BP/PCV-2, PCV-2 and control group during day $21 \sim 42(\mathrm{P}<0.05)$. Generally, IgG2a and IgG2b antibodies are related with Th1-type immune response and IgG1 antibodies are mediated Th2-type immune response. ${ }^{36}$ As shown in Figure $7 \mathrm{C}$, on days 28, 35, and42 after primary immunization, CYPP-PEI/PCV-2 group produced significantly higher levels of IgG1 (Th2-associated isotype) compared with other control groups except for ISA-206/PCV-2 group ( $\mathrm{P}<0.05)$. In addition, the highest levels of $\operatorname{IgG} 2 \mathrm{a}$ and IgG2b (Th2-associated isotype) were showed in CYPP-PEI /PCV-2 group on days 28, 35, and 42 after primary immunization (Figure 7D and E). However, there were not statistically significant differences of IgG2a levels between CYPP-PEI /PCV-2, CYPP/PCV-2, BP/PCV-2, and CYP/PCV-2 on day $28(\mathrm{P}>0.05)$. At the same time, IgG2b levels in CYPP-PEI /PCV-2 and CYPP/PCV-2 did not show significant difference on days 35 and 42 .

\section{Serum Cytokine Level in Mice}

To further evaluate the types of immune response induced by CYPP-PEI nanoparticles, cytokines were measured by ELISA on day 21and 35 after primary immunization. CYPP-PEI /PCV-2 induced significant secretion of IFN- $\gamma$ and TNF- $\alpha$ (Th1-type cytokines) compared with other control groups
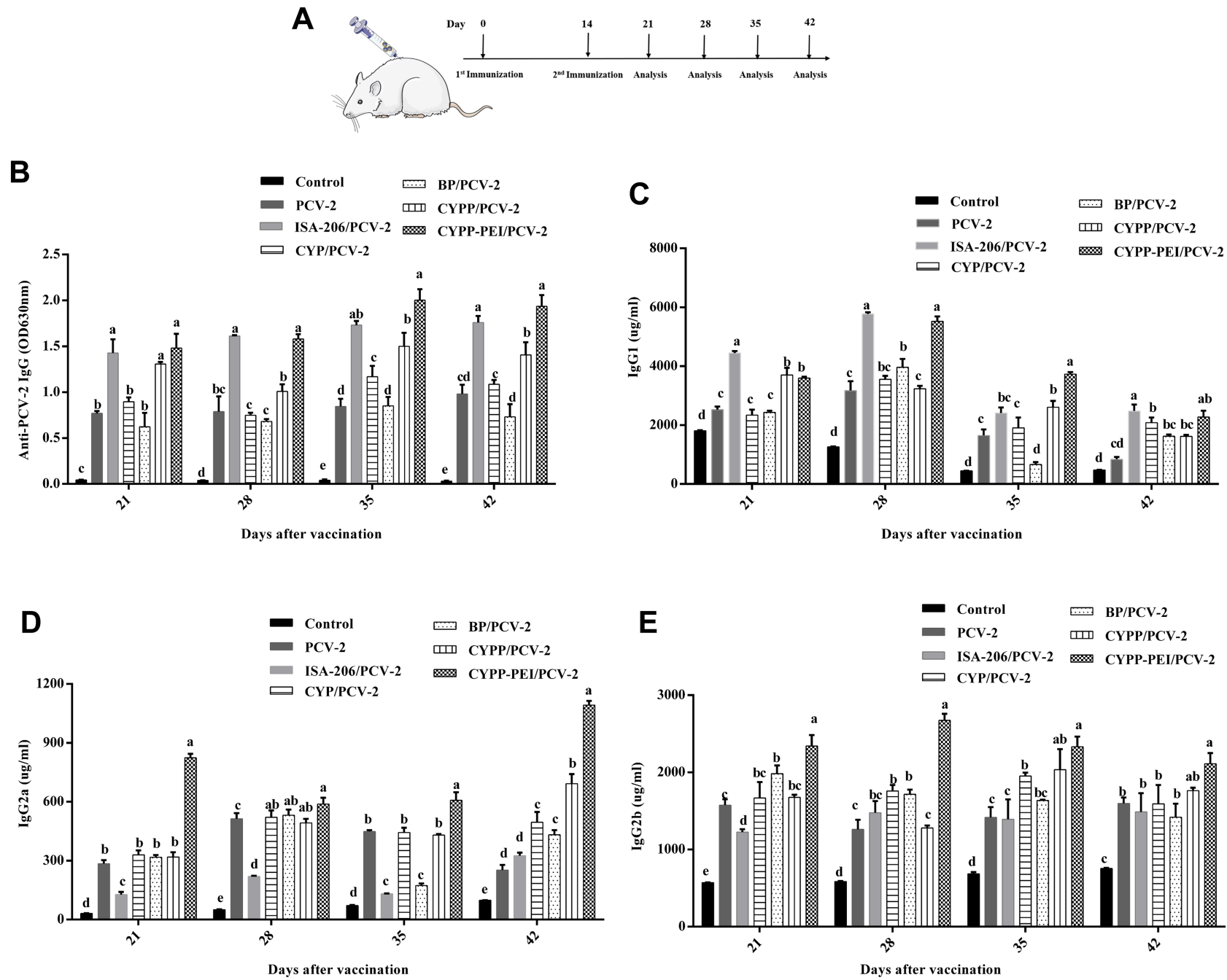

Figure 7 The levels of PCV-2-specific lgG and isotypes after immunized with different vaccine formulations. (A) Experimental scheme for the vaccination of mice. Mice were injected subcutaneously with $200 \mu \mathrm{l}$ of different vaccine formulation (ISA206/PCV-2, PCV-2 and PBS were used as control) on day 0 and day I4. (B) The serum expressions of PCV-2-specific IgG, isotypes of IgG (C) IgGI, (D) IgG2a, and (E) IgG2b were measured on day $21,28,35$, and 42 by ELISA Kits. Results were expressed as mean \pm SEM ( $n$ $=4)$. a-e Bars in the figure without the same superscripts differed significantly $(P<0.05)$. 
except for ISA-206/PCV-2 group on day 21 and $35(\mathrm{P}<0.05)$ (Figure $8 \mathrm{~A}$ and $\mathrm{B}$ ), which indicated that positive charged nanoparticles CYPP-PEI enhanced the secretion of Th1-type cytokines. In addition, the content of IL-4 and IL-6 (Th2-type cytokines) in serum was detected. In Figure 8C, the levels of IL-4 in CYPP-PEI/PCV-2 group were significantly higher than that in other groups on day $21(\mathrm{P}<0.05)$, while there was no obvious difference of IL-4 expression among CYPP-PEI/PCV2, CYPP/PCV-2 and ISA-206/PCV-2 on day 35 (P>0.05). As shown in Figure 8D, CYPP-PEI/PCV-2 significantly increased the expression of IL- 6 on day 21 and day 35 compared with CYP/PCV-2, BP/PCV-2, CYP/PCV-2, PCV-2, and control group $(\mathrm{P}<0.05)$.

\section{Discussion}

PLGA nanoparticles have advantages on controlling release in drug and vaccine delivery systems, which have attracted wide attention from biomedical application field. ${ }^{37} \mathrm{~A}$ common way
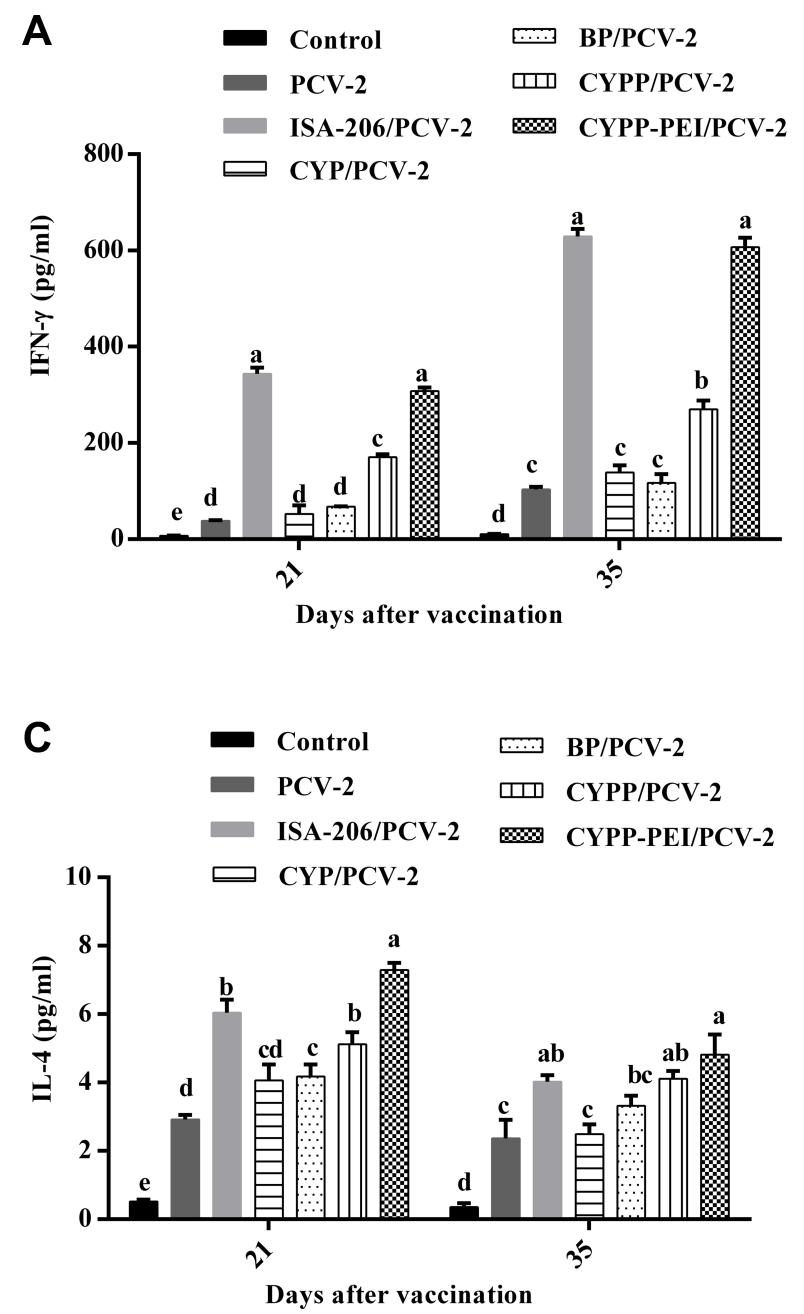

to make up the intrinsic immunogenic deficiency of some antigens is encapsulating both immunomodulators and antigens into PLGA shells. ${ }^{38}$ PLGA nanoparticles caused an antigen depot effect in the injection site, prolonged the release time of the antigen or adjuvant, and then induced strong and durable immune responses. ${ }^{39}$ Previous study had found that after encapsulating CYP into PLGA (CYPP), CYPP nanoparticles showed strong immunoenhancement activities. ${ }^{9}$ Moreover, nanoparticles which positively charged on the surfaces contributed to binding negatively charged APCs by electrostatic attraction, which could enhance the phagocytosis of APCs and induce stronger immune responses. $15,39,40$ Polyethylenimine (PEI), one of the common cationic polymers, has been extensively used to modify PLGA nanoparticles. ${ }^{24,41}$ In this study, in order to improve the efficacy of immunomodulator, a new antigen and immunomodulators co-delivery system was designed by encapsulated CYP in PLGA nanoparticles and modified with PEI (CYPP-PEI).
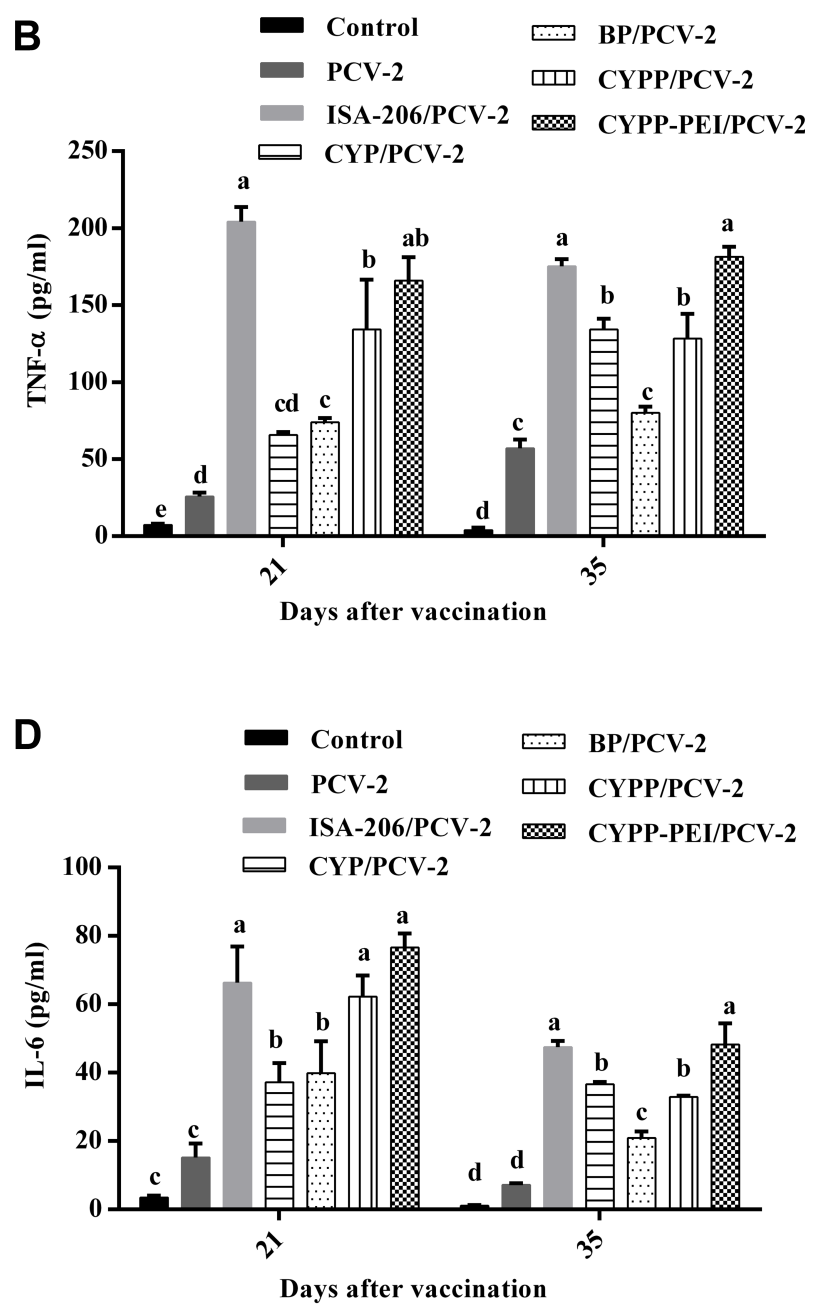

Figure 8 The content of cytokines in serum on day 2 I and day 28. (A) IFN- $\gamma$, (B) TNF- $\alpha$, (C) IL-4, and (D) IL-6 were measured by ELISA Kits. Results were expressed as mean $\pm \operatorname{SEM}(n=3)$. a-e Bars in the figure without the same superscripts differed significantly $(P<0.05)$. 
The preparation conditions of CYPP-PEI nanoparticles were optimized: the surface modification (the weight ratio of PLGA: PEI $=20: 1)$, average size $(326.6 \mathrm{~nm})$, zeta potential $(+41.5 \mathrm{mV})$, PDI (0.149), and encapsulation efficiency (EE) $(59 \pm 2.3 \%)$, for example. Both nanoparticles and PCV-2 absorbed nanoparticles showed excellent stabilities at $4^{\circ} \mathrm{C}$ for 1 month. In the meantime, absorption efficiency of CYPP-PEI/PCV-2 to antigens was higher than other groups. The modification of PEI increased the surface charge of nanoparticles, further contributed to the electrostatic interaction between the positive charged nanoparticles and the negative charged protein. ${ }^{42}$

Macrophages are antigen-presenting cells and play an important role in regulating immune responses. ${ }^{43}$ The phagocytosis of macrophages is essential to defend infections. ${ }^{30}$ The data showed that CYPP-PEI nanoparticles significantly enhanced the cellular uptake capacity of macrophages compared with other groups $(\mathrm{P}<0.05)$ (Figure 4A and $\mathrm{B}$ ). In addition, the CLSM images of nanoparticles in CYPP-PEI group reflected stronger green fluorescence (OVA-FITC) in macrophages than which in CYPP, BP, CYP, and control groups (Figure 4C). The PEI-modified nanoparticles exhibited positive surface charge and high antigen-absorbed efficiency, which might be beneficial to antigen uptake. After macrophages were activated, a large number of bioactive molecules were induced, such as costimulatory molecules and cytokines. ${ }^{44}$ Antigen-presenting cells upregulated the expression of costimulatory molecules (CD80, CD86) and then induced the activation of T-cell. ${ }^{45}$ As shown in Figure 5AC, the expressions of CD80 and CD86 costimulatory molecules in CYPP-PEI nanoparticles group were significantly upregulated, which suggested the activation of macrophages was enhanced by CYPP-PEI nanoparticles. Then, CYPP-PEI nanoparticles significantly increased the secretion of IFN- $\gamma$, IL-12p70, and TNF- $\alpha$ (Figure 5DF) under the action of activated macrophages. These results indicated that positively charged CYPP-PEI nanoparticles could promote macrophage activation and induce large amounts of cytokines.

Adjuvants or antigens carried by nanoparticle delivery systems could improve the targeting of DLNs and activate LN-resident DCs which was essential for initiating adaptive immunity. ${ }^{35}$ In Figure 6, after subcutaneous injection of vaccine formulation for $24 \mathrm{~h}$, the surface activation markers MHCI, MHCII, and CD80 were highly expressed in CD11 c+ cells treated with CYPP-PEI/PCV-2. In particular, the expressions of surface activation markers MHCI, MHCII, and CD80 in CYPP-PEI/PCV-2 group were significantly higher than ISA-206/PCV-2 group ( $\mathrm{P}>0.05$ ). Lower expression of the surface activation markers may attribute to the large size of the ISA-206/PCV-2 group, the oversized particles could not be delivered to the dendritic cells in $24 \mathrm{~h}$ after injection. ${ }^{46}$ These results indicated that positively charged CYPP-PEI nanoparticles could promote both PCV-2 antigen and CYP immunomodulator delivery to DCs in lymph nodes. What's more, it could further enhance the activity of CYP and activate DCs more efficiently.

Improving the immunogenicity of PCV-2 vaccine with immune adjuvant is an effective strategy to reduce the huge economic losses caused by PCV-2 in the swine industry. ${ }^{20}$ Aluminum salt adjuvant and oil emulsion adjuvant have been widely used in veterinary medicine, but these adjuvants could cause some local irritation effects, such as injection site pain, inflammatory response, and tissue damage. ${ }^{47,48}$ Therefore, CYPP-PEI nanoparticles were selected as a safe and effective adjuvant to absorb PCV-2 antigen and improve immune response. Compared with CYPP/PCV-2, CYP/PCV2, BP/PCV-2, PCV-2, and control groups, CYPP-PEI/PCV-2 induced significantly strong and persistent $\mathrm{PCV}$-2-specific IgG (Figure 7B). These results demonstrated CYPP-PEI nanoparticles were effective adjuvant to induce high PCV2-specific IgG antibodies. Meanwhile, Th2-type antibody of IgG1 and Th1-type antibodies of IgG2a and IgG2b showed obviously increased with CYPP-PEI/PCV-2 in serum (Figure 7CE). Cytokines, important immune regulatory molecules, are critical for identifying the type of immune response. ${ }^{49}$ Th1-type cytokines (IFN- $\gamma$, TNF-a) are key effectors of the inflammatory response and correlated with cellular response. Th2-type cytokines (IL-4, IL-6) play important roles in humoral response. ${ }^{50-53}$ In Figure 8, CYPP$\mathrm{PEI} / \mathrm{PCV}-2$ significantly promoted the levels of IFN-r, TNF-a (Th1-type cytokines), IL-4, and IL-6 (Th2-type cytokines), which indicated that CYPP-PEI nanoparticles could promote both Th1-type and Th2-type immune response. Although the content of Th2-type cytokines (IL-4, IL-6) in the CYPP-PEI group increased obviously compared with PCV-2, CYP/ PCV-2, BP/PCV-2, and control groups, their expression levels were significantly lower than Th1-type cytokines. These results suggested that CYPP-PEI/PCV-2 stimulated the secretion of Th1 and Th2 type cytokines and cationicmodified nanoparticles with PCV-2 absorbed on the surface (CYPP/PCV-2) could promote a Th1/Th2 mixed but Th1biased immune responses. Thus, positively charged CYPPPEI was the potential antigen delivery system to promote the immune response and resist infectious diseases. 


\section{Conclusion}

In conclusion, the present study revealed that cationic polymer PEI-modified CYP-encapsulated PLGA nanoparticles could advance antigen absorption efficiency, promote antigen uptake by macrophages, and enhance macrophage activation. Moreover, the positively charged CYPP-PEI nanoparticles were benefit to deliver antigen and CYP immunomodulator into lymph nodes and activate DCs, and further enhance Th1/Th2 mixed but Th1-biased immune responses in vivo. Generally speaking, positive charged CYPP-PEI nanoparticles showed an outstanding application prospect as a novel vaccine adjuvant.

\section{Abbreviations}

PLGA, Poly(lactic-co-glycolic acid); CYP, Chinese yam polysaccharide; $\mathrm{PEI}$, polyethylenimine; APCs, antigenpresenting cells; PCV-2, porcine circovirus type 2; DCs, dendritic cells; DLNs, draining lymph nodes; F68, Pluronic F68; TEM, transmission electron microscopy; PDI, polydispersity index; EE, encapsulation efficiency; BCA, bicinchoninic acid; OVA-FITC, fluorescein isothiocyanate labeled ovalbumin; AR, adsorption rate; HRP, horseradish peroxidase.

\section{Funding}

The project was supported by the National Natural Science Foundation of China (Grant No. 31872509, 31672596), the Fundamental Research Funds for the Central Universities (Grant No. KYZ201844), and A Project Funded by the Priority Academic Program Development of Jiangsu Higher Education Institutions (PAPD). We are grateful to all of the other staff members at the Institute of Traditional Chinese Veterinary Medicine of Nanjing Agricultural University for their assistance in this study.

\section{Disclosure}

The authors report no conflicts of interest in this work.

\section{References}

1. Yang W, Wang Y, Li X, Yu P. Purification and structural characterization of Chinese yam polysaccharide and its activities. Carbohydr Polym. 2015;117:1021-1027. doi:10.1016/j.carbpol.2014.09.082

2. Choi EM, Koo SJ, Hwang JK. Immune cell stimulating activity of mucopolysaccharide isolated from yam (Dioscorea batatas). J Ethnopharmacol. 2004;91(1):1-6. doi:10.1016/j.jep.2003.11.006

3. Kong XF, Zhang YZ, Yin YL, et al. Chinese yam polysaccharide enhances growth performance and cellular immune response in weanling rats. J Sci Food Agric. 2009;89(12):2039-2044. doi:doi/10.1002/ jsfa. 3688
4. Xue HY, Li JR, Liu YG, et al. Optimization of the ultrafiltration-assisted extraction of Chinese yam polysaccharide using response surface methodology and its biological activity. Int $J$ Biol Macromol. 2019;121:1186-1193. doi:10.1016/j.ijbiomac.2018.10.126

5. Zhi F, Yang TL, Wang Q, et al. Isolation, structure and activity of a novel water-soluble polysaccharide from Dioscorea opposita Thunb. Int J Biol Macromol. 2019;133:1201-1209. doi:10.1016/j. ijbiomac.2019.04.087

6. Shi Y, Lian YU, Zhai M, et al. Effect of Nano Chinese Yam Polysaccharide on 4 kinds of tumor cells. Chinese $j$ Modern Applied Pharm. 2016.

7. Zhao GH, Zhi-Xiao LI, Chen ZDJAPS. Structural analysis and antitumor activity of RDPS-I polysaccharide from Chinese yam. Acta Pharmaceutica Sinica. 2003;38(1):37-41. doi:10.1023/A:1022865 704606

8. Li M, Chen L-X, Chen S-R, et al. Non-starch polysaccharide from Chinese yam activated RAW 264.7 macrophages through the Toll-

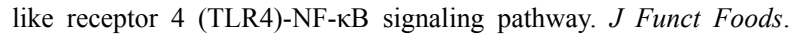
2017;37:491-500. doi:10.1016/j.jff.2017.08.025

9. Luo L, Qin T, Huang Y, et al. Exploring the immunopotentiation of Chinese yam polysaccharide poly(lactic-co-glycolic acid) nanoparticles in an ovalbumin vaccine formulation in vivo. Drug Deliv. 2017;24(1):1099-1111. doi:10.1080/10717544.2017. 1359861

10. Chowhan A, Giri TK. Polysaccharide as renewable responsive biopolymer for in situ gel in the delivery of drug through ocular route. Int J Biol Macromol. 2020;150:559-572. doi:10.1016/j.ijbiomac. 2020.02.097

11. Makadia HK, Siegel SJ. Poly Lactic-co-Glycolic Acid (PLGA) as biodegradable controlled drug delivery carrier. Polymers. 2011;3 (3):1377-1397. doi:10.3390/polym3031377

12. Xu Y, Kim CS, Saylor DM, Koo D. Polymer degradation and drug delivery in PLGA-based drug-polymer applications: A review of experiments and theories. J Biomed Mater Res B Appl Biomater. 2017;105(6):1692-1716. doi:10.1002/jbm.b.33648

13. Jiang W, Gupta RK, Deshpande MC, Schwendeman SP. Biodegradable poly(lactic-co-glycolic acid) microparticles for injectable delivery of vaccine antigens. Adv Drug Deliv Rev. 2005;57 (3):391-410. doi:10.1016/j.addr.2004.09.003

14. Hafner AM, Corthesy B, Textor M, Merkle HP. Surface-assembled poly(I:C) on PEGylated PLGA microspheres as vaccine adjuvant: APC activation and bystander cell stimulation. Int J Pharm. 2016;514(1):176-188. doi:10.1016/j.ijpharm.2016.07.042

15. Huh MS, Lee SY, Park S, et al. Tumor-homing glycol chitosan/ polyethylenimine nanoparticles for the systemic delivery of siRNA in tumor-bearing mice. J Control Release. 2010;144(2):134-143. doi:10.1016/j.jconrel.2010.02.023

16. Wusiman A, He J, Zhu T, et al. Macrophage immunomodulatory activity of the cationic polymer modified PLGA nanoparticles encapsulating Alhagi honey polysaccharide. Int $J$ Biol Macromol. 2019;134:730-739. doi:10.1016/j.ijbiomac.2019.05.038

17. Lu B, Lv X, Le Y. Chitosan-Modified PLGA nanoparticles for control-released drug delivery. Polymers. 2019;11:2. doi:10.3390/ polym11020304

18. Bivas-Benita M, Lin MY, Bal SM, et al. Pulmonary delivery of DNA encoding Mycobacterium tuberculosis latency antigen Rv1733c associated to PLGA-PEI nanoparticles enhances $\mathrm{T}$ cell responses in a DNA prime/protein boost vaccination regimen in mice. Vaccine. 2009;27(30):4010-4017. doi:10.1016/j.vaccine. 2009.04.033

19. Rose N, Opriessnig T, Grasland B, Jestin A. Epidemiology and transmission of porcine circovirus type 2 (PCV2). Virus Res. 2012;164(12):78-89. doi:10.1016/j.virusres.2011.12.002

20. Guo X, Zheng Q, Jiang X, et al. The composite biological adjuvants enhance immune response of porcine circovirus type2 vaccine. Vet Microbiol. 2019;228:69-76. doi:10.1016/j.vetmic.2018.11.015 
21. Zhang G, Jia P, Cheng G, et al. Enhanced immune response to inactivated porcine circovirus type 2 (PCV2) vaccine by conjugation of chitosan oligosaccharides. Carbohydr Polym. 2017;166:64-72. doi:10.1016/j.carbpol.2017.02.058

22. Schepetkin IA, Quinn MT. Botanical polysaccharides: macrophage immunomodulation and therapeutic potential. Int Immunopharmacol. 2006;6(3):317-333. doi:10.1016/j.intimp.2005.10.005

23. Yu Q, Nie SP, Wang JQ, Yin PF, Li WJ, Xie MY. Polysaccharide from Ganoderma atrum induces tumor necrosis factor-alpha secretion via phosphoinositide 3-kinase/Akt, mitogen-activated protein kinase and nuclear factor-kappaB signaling pathways in RAW264.7 cells. Int Immunopharmacol. 2012;14(4):362-368. doi:10.1016/j.intimp. 2012.09.005

24. Mulens-Arias V, Rojas JM, Perez-Yague S, Morales MP, Barber DF. Polyethylenimine-coated SPIONs trigger macrophage activation through TLR-4 signaling and ROS production and modulate podosome dynamics. Biomaterials. 2015;52:494-506. doi:10.1016/j. biomaterials.2015.02.068

25. Cruz LJ, Tacken PJ, Fokkink R, et al. Targeted PLGA nano- but not microparticles specifically deliver antigen to human dendritic cells via DC-SIGN in vitro. $J$ Control Release. 2010;144(2):118-126. doi:10.1016/j.jconrel.2010.02.013

26. Roopngam P. Poly (Lactic-Co-Glycolic) Acid (PLGA) adjuvant for immunotherapy. Immunol Disord Immunother. 2017;2:113. doi:10.35248/2593-8509.17.2.113

27. Sahiner N, Sagbas S, Sahiner M, Ayyala RS. Polyethyleneimine modified poly(Hyaluronic acid) particles with controllable antimicrobial and anticancer effects. Carbohydr Polym. 2017;159:29-38. doi:10.1016/j.carbpol.2016.12.024

28. Chen X, Liu Y, Wang L, et al. Enhanced humoral and cell-mediated immune responses generated by cationic polymer-coated PLA microspheres with adsorbed HBsAg. Mol Pharm. 2014;11(6):1772-1784. doi:10.1021/mp400597z

29. Luo L, Zheng S, Huang Y, et al. Preparation and characterization of Chinese yam polysaccharide PLGA nanoparticles and their immunological activity. Int J Pharm. 2016;511(1):140-150. doi:10.1016/j. ijpharm.2016.06.130

30. Bo R, Ma X, Feng Y, et al. Optimization on conditions of Lycium barbarum polysaccharides liposome by RSM and its effects on the peritoneal macrophages function. Carbohydr Polym. 2015;117:215-222. doi:10.1016/j.carbpol.2014.09.060

31. Xu S, Wusiman A, Liu Z, et al. pH-responsive Astragalus polysaccharides-loaded poly(lactic-co-glycolic acid) nanoparticles and their in vitro immunogenicity. Int $J$ Biol Macromol. 2019;125:865-875. doi:10.1016/j.ijbiomac.2018.12.156

32. Qian Y, Liang X, Yang J, et al. Hyaluronan reduces cationic liposome-induced toxicity and enhances the antitumor effect of targeted gene delivery in mice. ACS Appl Mater Interfaces. 2018;10 (38):32006-32016. doi:10.1021/acsami.8b12393

33. Lu Y, Yang Y, Gu Z, et al. Glutathione-depletion mesoporous organosilica nanoparticles as a self-adjuvant and Co-delivery platform for enhanced cancer immunotherapy. Biomaterials. 2018;175:82-92. doi:10.1016/j.biomaterials.2018.05.025

34. Godbey WT, Wu KK, Release A. Poly(ethylenimine) and its role in gene delivery. J Control Release. 1999;60(23):149-160. doi:10.1016/ s0168-3659(99)00090-5

35. Jeanbart L, Ballester M, de Titta A, et al. Enhancing efficacy of anticancer vaccines by targeted delivery to tumor-draining lymph nodes. Cancer Immunol Res. 2014;2(5):436-447. doi:10.1158/23266066.Cir-14-0019-t

36. Ebrahimian M, Hashemi M, Maleki M, et al. Induction of a balanced Th1/Th2 immune responses by co-delivery of PLGA/ovalbumin nanospheres and $\mathrm{CpG}$ ODNs/PEI-SWCNT nanoparticles as TLR9 agonist in BALB/c mice. Int $J$ Pharm. 2016;515(12):708-720. doi:10.1016/j.ijpharm.2016.10.065
37. Akagi T, Baba M, Akashi M. Biodegradable nanoparticles as vaccine adjuvants and delivery systems: regulation of immune responses by nanoparticle-based vaccine. Adv Adv Polymer Sci. 2012. doi:10.1007/ 12_2011_150

38. Silva AL, Soema PC, Slutter B, Ossendorp F, Jiskoot W. PLGA particulate delivery systems for subunit vaccines: linking particle properties to immunogenicity. Hum Vaccin Immunother. 2016;12 (4):1056-1069. doi:10.1080/21645515.2015.1117714

39. Zhang W, Wang L, Liu Y, et al. Immune responses to vaccines involving a combined antigen-nanoparticle mixture and nanoparticleencapsulated antigen formulation. Biomaterials. 2014;35 (23):6086-6097. doi:10.1016/j.biomaterials.2014.04.022

40. Skwarczynski M, Toth I. Recent advances in peptide-based subunit nanovaccines. Nanomedicine. 2014;9(17):2657-2669. doi:10.2217/ nnm.14.187

41. Seok H, Noh JY, Lee DY, Kim SJ, Song CS, Kim YC. Effective humoral immune response from a H1N1 DNA vaccine delivered to the skin by microneedles coated with PLGA-based cationic nanoparticles. J Control Release. 2017;265:66-74. doi:10.1016/j. jconrel.2017.04.027

42. Zupancic E, Curato C, Paisana M, et al. Rational design of nanoparticles towards targeting antigen-presenting cells and improved $\mathrm{T}$ cell priming. J Control Release. 2017;258:182-195. doi:10.1016/j. jconrel.2017.05.014

43. Wang Z, Liu Z, Zhou L, Long T, Zhou X, Bao Y. Immunomodulatory effect of APS and PSP is mediated by Ca2(+)-cAMP and TLR4/ NF-kappaB signaling pathway in macrophage. Int J Biol Macromol. 2017;94(PtA):283-289. doi:10.1016/j.ijbiomac.2016.10.018

44. Lu -X-X, Jiang Y-F, Li H, et al. Polymyxin B as an inhibitor of lipopolysaccharides contamination of herb crude polysaccharides in mononuclear cells. Chin J Nat Med. 2017;15(7):487-494. doi:10.1016/s1875-5364(17)30074-2

45. Inaba K, Inaba M. Antigen recognition and presentation by dendritic cells. Int J Hematol. 2005;81(3):181-187. doi:10.1532/IJH97.04200

46. Kim SY, Noh YW, Kang TH, et al. Synthetic vaccine nanoparticles target to lymph node triggering enhanced innate and adaptive antitumor immunity. Biomaterials. 2017;130:56-66. doi:10.1016/j. biomaterials.2017.03.034

47. Oreskovic Z, Kudlackova H, Krejci J, Nechvatalova K, Faldyna M. Oil-based adjuvants delivered intradermally induce high primary IgG2 immune response in swine. Res Vet Sci. 2017;114:41-43. doi:10.1016/j.rvsc.2017.03.007

48. Petrovsky N. Comparative safety of vaccine adjuvants: a summary of current evidence and future needs. Drug Saf. 2015;38 (11):1059-1074. doi:10.1007/s40264-015-0350-4

49. Singh D, Somani VK, Aggarwal S, Bhatnagar R. PLGA (85:15) nanoparticle based delivery of $\mathrm{rL7/L12}$ ribosomal protein in mice protects against Brucella abortus 544 infection: A promising alternate to traditional adjuvants. Mol Immunol. 2015;68(2Pt A):272-279. doi:10.1016/j.molimm.2015.09.011

50. Hu Y, He K, Wang X. Role of Chinese herbal medicinal ingredients in secretion of cytokines by PCV2-induced endothelial cells. J Immunotoxicol. 2016;13(2):141-147. doi:10.3109/1547691X.2015. 1017624

51. Wusiman A, Xu S, Ni H, et al. Immunomodulatory effects of Alhagi honey polysaccharides encapsulated into PLGA nanoparticles. Carbohydr Polym. 2019;211:217-226. doi:10.1016/j.carbpol.2019. 01.102

52. Malik A, Gupta M, Mani R, Bhatnagar R. Single-dose Ag85B-ESAT6loaded poly(lactic-co-glycolic acid) nanoparticles confer protective immunity against tuberculosis. Int $J$ Nanomedicine. 2019;14:3129-3143. doi:10.2147/IJN.S172391

53. Manish M, Rahi A, Kaur M, Bhatnagar R, Singh S. A single-dose PLGA encapsulated protective antigen domain 4 nanoformulation protects mice against Bacillus anthracis spore challenge. PLoS One. 2013;8(4):e61885. doi:10.1371/journal.pone.0061885 


\section{Publish your work in this journal}

The International Journal of Nanomedicine is an international, peerreviewed journal focusing on the application of nanotechnology in diagnostics, therapeutics, and drug delivery systems throughout the biomedical field. This journal is indexed on PubMed Central, MedLine, CAS, SciSearch ${ }^{\mathbb{B}}$, Current Contents ${ }^{\mathbb{B}} /$ Clinical Medicine, $^{2}$
Journal Citation Reports/Science Edition, EMBase, Scopus and the Elsevier Bibliographic databases. The manuscript management system is completely online and includes a very quick and fair peer-review system, which is all easy to use. Visit http://www.dovepress.com/ testimonials.php to read real quotes from published authors.

Submit your manuscript here: https://www.dovepress.com/international-journal-of-nanomedicine-journal 